\title{
THE RECENT STAR FORMATION IN NGC 6822: AN ULTRAVIOLET STUDY
}

\author{
Boryana V. Efremova ${ }^{1}$, Luciana Bianchi ${ }^{1}$, David A. Thilker ${ }^{1}$, James D. Neill $^{2}$, Denis Burgarella $^{3}$, Ted K. Wyder ${ }^{2}$, \\ Barry F. Madore ${ }^{4}$, Soo-Chang Rey ${ }^{5,6}$, Tom A. Barlow ${ }^{2}$, Tim Conrow ${ }^{2}$, Karl Forster $^{2}$, Peter G. Friedman $^{2}$, \\ D. Christopher Martin ${ }^{2}$, Patrick Morrissey ${ }^{2}$, Susan G. NefF $^{6}$, David Schiminovich $^{7}$, Mark Seibert $^{4}$, and Todd Small ${ }^{2}$ \\ ${ }^{1}$ Department of Physics \& Astronomy, Johns Hopkins University, 3400 North Charles Street, Baltimore, MD 21218, USA; \\ boryana@pha.jhu.edu,bianchi@pha.jhu.edu \\ ${ }^{2}$ California Institute of Technology, MC 278-17, 1200 East California Boulevard, Pasadena, CA 91125, USA \\ ${ }^{3}$ Laboratoire d'Astrophysique de Marseille, BP 8, Traverse du Siphon, 13376 Marseille Cedex 12, France \\ ${ }^{4}$ Observatories of the Carnegie Institution of Washington, 813 Santa Barbara Street, Pasadena, CA 91101, USA \\ ${ }^{5}$ Department of Astronomy and Space Science, Chungnam National University, Daejeon 305-764, Republic of Korea \\ ${ }^{6}$ Laboratory for Astronomy and Solar Physics, NASA Goddard Space Flight Center, Greenbelt, MD 20771, USA \\ 7 Department of Astronomy, Columbia University, New York, NY 10027, USA \\ Received 2009 April 19; accepted 2011 January 7; published 2011 March 9
}

\begin{abstract}
We characterize the star formation in the low-metallicity galaxy NGC 6822 over the past few hundred million

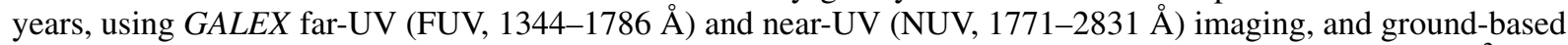
$\mathrm{H} \alpha$ imaging. From the GALEX FUV image, we define 77 star-forming (SF) regions with area $>860 \mathrm{pc}^{2}$, and surface brightness $\lesssim 26.8 \mathrm{mag}(\mathrm{AB}) \operatorname{arcsec}^{-2}$, within $0.2(1.7 \mathrm{kpc})$ of the center of the galaxy. We estimate the extinction by interstellar dust in each SF region from resolved photometry of the hot stars it contains: $E(B-V)$ ranges from the minimum foreground value of $0.22 \mathrm{mag}$ up to $0.66 \pm 0.21 \mathrm{mag}$. The integrated FUV and NUV photometry, compared with stellar population models, yields ages of the SF complexes up to a few hundred Myr, and masses from $2 \times 10^{2} \mathcal{M}_{\odot}$ to $1.5 \times 10^{6} \mathcal{M}_{\odot}$. The derived ages and masses strongly depend on the assumed type of interstellar selective extinction, which we find to vary across the galaxy. The total mass of the FUV-defined SF regions translates into an average star formation rate (SFR) of $1.4 \times 10^{-2} \mathcal{M}_{\odot} \mathrm{yr}^{-1}$ over the past $100 \mathrm{Myr}$, and $\mathrm{SFR}=1.0 \times 10^{-2} \mathcal{M}_{\odot} \mathrm{yr}^{-1}$ in the most recent $10 \mathrm{Myr}$. The latter is in agreement with the value that we derive from the $\mathrm{H} \alpha$ luminosity, SFR $=0.008 \mathcal{M}_{\odot} \mathrm{yr}^{-1}$. The SFR in the most recent epoch becomes higher if we add the $\mathrm{SFR}=0.02 \mathcal{M}_{\odot} \mathrm{yr}^{-1}$ inferred from far-IR measurements, which trace star formation still embedded in dust (age $\lesssim$ a few Myr).
\end{abstract}

Key words: galaxies: individual (NGC 6822) - galaxies: stellar content - Local Group - stars: formation ultraviolet: stars

\section{INTRODUCTION}

Continuum fluxes in the ultraviolet (UV) and infrared (IR) spectral regions, and $\mathrm{H} \alpha$ line emission, are the main indicators of star formation activity in distant galaxies (see, e.g., Kennicutt 1998). The UV flux is a direct tracer of young massive stars, whose energy is mostly emitted in this spectral region, $\mathrm{H} \alpha$ emission originates from interstellar gas ionized by the most massive stars, and the far-IR emission is produced by dust particles re-emitting reprocessed UV stellar light.

Integrated measurements of these fluxes can be translated into star formation rates (SFRs) of galaxies, but additional information is needed. First, observed fluxes need to be corrected for extinction by interstellar dust, both foreground (by Milky Way (MW) dust along the line of sight) and internal (within the galaxy). Reddening is particularly significant at UV wavelengths (see, e.g., Bianchi 2011). Stellar population models with adequate star formation history (SFH) are then used to transform the continuum and line-emission luminosities into SFRs.

The UV photometry of SF galaxies is usually corrected for interstellar extinction assuming an MW-type selective extinction with $R_{V}=3.1$ (Cardelli et al. 1989) for the foreground component, and the Calzetti (2001) extinction curve for internal extinction. The amount of extinction is sometimes estimated by comparison of UV and far-IR fluxes (Calzetti et al. 2005; Cortese et al. 2006; Boissier et al. 2007; Meurer et al. 2009). Such method assumes that the intrinsic FUV-NUV color is known, however, its value strongly varies with age for young starbursts (e.g., Bianchi 2009, 2011), and that UV and far-IR fluxes are emitted by the same population, which is often not the case.

In unresolved distant galaxies only integrated measurements are possible, and a global extinction correction and SFH must be assumed for interpreting such measurements. On the other hand, in nearby galaxies individual SF regions can be measured, and their stellar content studied in detail (e.g., Bianchi \& Efremova 2006; Bianchi et al. 2001, 2010, 2011; Kang et al. 2009 , and references therein). Therefore, the dust properties can be explored in a variety of local environments, providing information on the interplay of dust and star formation, and a calibration of star formation indicators in distant galaxies.

Deep imaging in FUV and NUV for hundreds of nearby galaxies was obtained with the Galaxy Evolution Explorer (GALEX; Martin et al. 2005; Morrissey et al. 2007) as part of the Nearby Galaxy Survey (NGS; Bianchi et al. 2003a, 2003b; Bianchi 2009; Gil de Paz et al. 2007). The wide-field UV imaging provides a characterization of the young stellar populations across the whole extent of these galaxies, and can be used, with complementary optical data, to infer their SFH and SFR.

In this paper, we perform a comprehensive study of the young stellar populations in the Local Group low-metallicity galaxy NGC 6822, the nearest SF galaxy currently with no massive neighbor. We identify and define SF regions from GALEX wide-field imaging in FUV, where the hottest, youngest 
stars are more prominent, throughout the extent of the galaxy. We use integrated photometry of these regions in FUV and $\mathrm{NUV}$, and complementary $\mathrm{H} \alpha$ emission-line imaging, as well as information from resolved stellar photometry, to investigate the star formation in this galaxy during the past $\sim 100 \mathrm{Myr}$, and the characteristics of interstellar extinction. The study of this galaxy, together with results for Local Group galaxies of other types, contributes one piece to a broader puzzle, aimed at understanding the modalities of star formation in differing environments, and the role of dust.

This benchmark galaxy was chosen to complement the study by Kang et al. (2009) of M31, and of other Local Group galaxies by Bianchi et al. (2010, 2011), because of its low metallicity and vicinity (494 kpc; McAlary et al. 1983) and the abundant information available from resolved stellar population studies with Hubble Space Telescope (HST) multi-band imaging (Bianchi et al. 2001; Bianchi \& Efremova 2006), CTIO (Massey et al. 2007b), Very Large Telescope (VLT) $U, B, V$ imaging, and extensive spectroscopy (B. V. Efremova et al. 2011, in preparation). NGC 6822's metallicity is believed to be subsolar: measurements by Muschielok et al. (1999) of three B-type supergiants and by Venn et al. (2001) of two A-type supergiants both yield $Z \approx 0.006$.

The paper is arranged as follows. In Section 2, we define SF regions from GALEX FUV imaging, and measure their integrated fluxes in FUV and NUV; we also use the Cerro Tololo Inter-American Observatory (CTIO) $\mathrm{H} \alpha$ imaging of Massey et al. (2007a) to define and measure regions of $\mathrm{H} \alpha$ emission. In Section 3, the integrated measurements of the SF regions are analyzed with stellar population models to derive their ages and masses, after the interstellar extinction is estimated from the massive stars within each SF region. The results are discussed in Section 4 and summarized in Section 5.

\section{OBSERVATIONS, DETECTION, AND PHOTOMETRY OF THE STAR-FORMING REGIONS}

\subsection{UV Imaging}

We used GALEX images in FUV $\left(\lambda_{\text {eff }}=1539 \AA\right.$, FWHM $\approx 270 \AA$ ), and NUV ( $\lambda_{\text {eff }}=2316 \AA$, FWHM $\approx 615 \AA$ ) with resolution 4".2 (FUV), and 5".3 (NUV; Morrissey et al. 2007), corresponding to $\sim 12 \mathrm{pc}$ at the distance of NGC 6822 . The images are sampled with $1^{\prime \prime} .5$ pixels.

The GALEX images of NGC 6822 were taken on 2005 August 20 as part of the NGS program, with exposure times of $4654 \mathrm{~s}$ (FUV) and $6198 \mathrm{~s}$ (NUV). The data were downloaded from the MAST archive. The 1.2 diameter GALEX field of view is centered at R.A. $=19^{\mathrm{h}} 44^{\mathrm{m}} 57^{\mathrm{s}} \cdot 37$, decl. $=-14^{\circ} 47^{\prime} 33^{\prime \prime} \cdot 32$, near the center of the galaxy (R.A. $=19^{\mathrm{h}} 44^{\mathrm{m}} 57.8$, decl. $=-14^{\circ} 48^{\prime} 11^{\prime \prime}$, FK5 2000). NGC 6822, with an optical diameter (at $\sim 25 \mathrm{mag} \operatorname{arcsec}^{-2}$ ) of 15'6 (Karachentsev et al. 2004 ), is contained in the central portion of the GALEX image, which is shown in Figure 1.

\subsection{UV Source Detection}

We identified the SF regions using the GALEX FUV image, which unambiguously reveals the young, hot massive stars not heavily embedded in interstellar dust. We followed the general method of Kang et al. (2009), adapted to the case of NGC 6822. We defined contours of regions with FUV surface brightness $\geqslant 3 \sigma$ above the background. An important issue in defining extended source contours and measuring their flux is the background estimate. Several approaches were used to find the best method for background evaluation (see also the discussion in Kang et al. 2009). For the purpose of source detection only, we constructed a background image applying a two-step circular median filter (64 pixels diameter, $\approx 1.5$ arcmin) to the FUV intensity map ("int" file). The first pass of the filter identifies pixels which belong to localized peaks via masking pixels brighter than the local median background estimate. The second pass of the filter operates only on the final list of non-peak pixels to obtain a background image less biased by substructure than a one-pass median filter. The diameter of the median filter was chosen to provide a background image where measurements of the background for individual sources are closest to the median flux density of the intensity map images in 6 pixel wide annuli around the sources, from here on the "local background." The background image produced using the adopted median filter gives sky estimates slightly lower (by about $0.17 / 0.18 \mathrm{mag} \operatorname{arcsec}^{-2}$ for FUV/NUV) than the local background. For comparison, the background image provided by the pipeline gives a background estimate lower than the local background by $0.52 / 0.60 \mathrm{mag} \operatorname{arcsec}^{-2}$ on average for FUV/NUV. A background-subtracted image was constructed, subtracting our background image from the intensity map image, and used for source detection.

Source contours were defined to enclose contiguous pixels with FUV flux more than $3 \sigma$ above the background image. This threshold corresponds to an FUV surface brightness of 0.0015 counts $\mathrm{s}^{-1}$ pixel $^{-1}\left(26.8 \mathrm{AB}\right.$ mag $\left.\operatorname{arcsec}^{-2}\right)$ on the background-subtracted image, or average 0.0025 counts $\mathrm{s}^{-1}$ pixel $^{-1}$ (26.2 AB mag $\operatorname{arcsec}^{-2}$ ) on the intensity map image.

The effect of the threshold choice on the source-contour definition is illustrated in the top panels of Figure 1, which show SF regions 75, 57, 27, and 20 defined for thresholds of $1 \sigma$ (green), $3 \sigma$ (light blue), and $4 \sigma$ (dark blue). A low threshold of $1 \sigma$ or $2 \sigma$ would cause regions such as 27 and 20 (OB8 and OB6, from here on "OB" designations are from Hodge 1977) to merge, and the main body of the galaxy to appear as one large region. If a threshold higher than $3 \sigma$ is used, sparse associations like region 75 (OB15) split into several sources or into individual stars (see also Kang et al. 2009 for more discussion on the procedure and the choice of parameters).

The sources defined using the $3 \sigma$ threshold follow the distribution of blue stars as shown by resolved stellar photometry from HST imaging (Bianchi et al. 2001; Bianchi \& Efremova 2006) and ground-based data (Massey et al. 2007b). To exclude artifacts, in the initial list we rejected sources with areas less than $16 \operatorname{arcsec}^{2}$ (7 pixels).

We further restricted the analysis sample to sources larger than $150 \operatorname{arcsec}{ }^{2}\left(\approx 860 \mathrm{pc}^{2}\right)$, in order to exclude single stars (mostly foreground) and background objects, and to examine SF complexes massive enough that stochastic effects will not be significant in deriving ages and masses by model analysis (Section 3). Stochasticity may affect the comparison of integrated star cluster photometry with stellar population models, as was first pointed out by Girardi et al. (1995). Quantitative assessment of this effect is still a matter of debate, given that more factors are relevant in such analysis, including initial mass function (IMF), metallicity, and extinction. For example, Fatuzzo \& Adams (2008) estimate that for clusters with $\gtrsim 1000$ stars the IMF is sampled well enough so that their UV flux is close to model predictions for integrated populations (but they also point out that the exact limit may vary with IFM). Their analysis concerns statistical distributions of bound, spherical, zero-age stellar clusters. Such limit corresponds to 


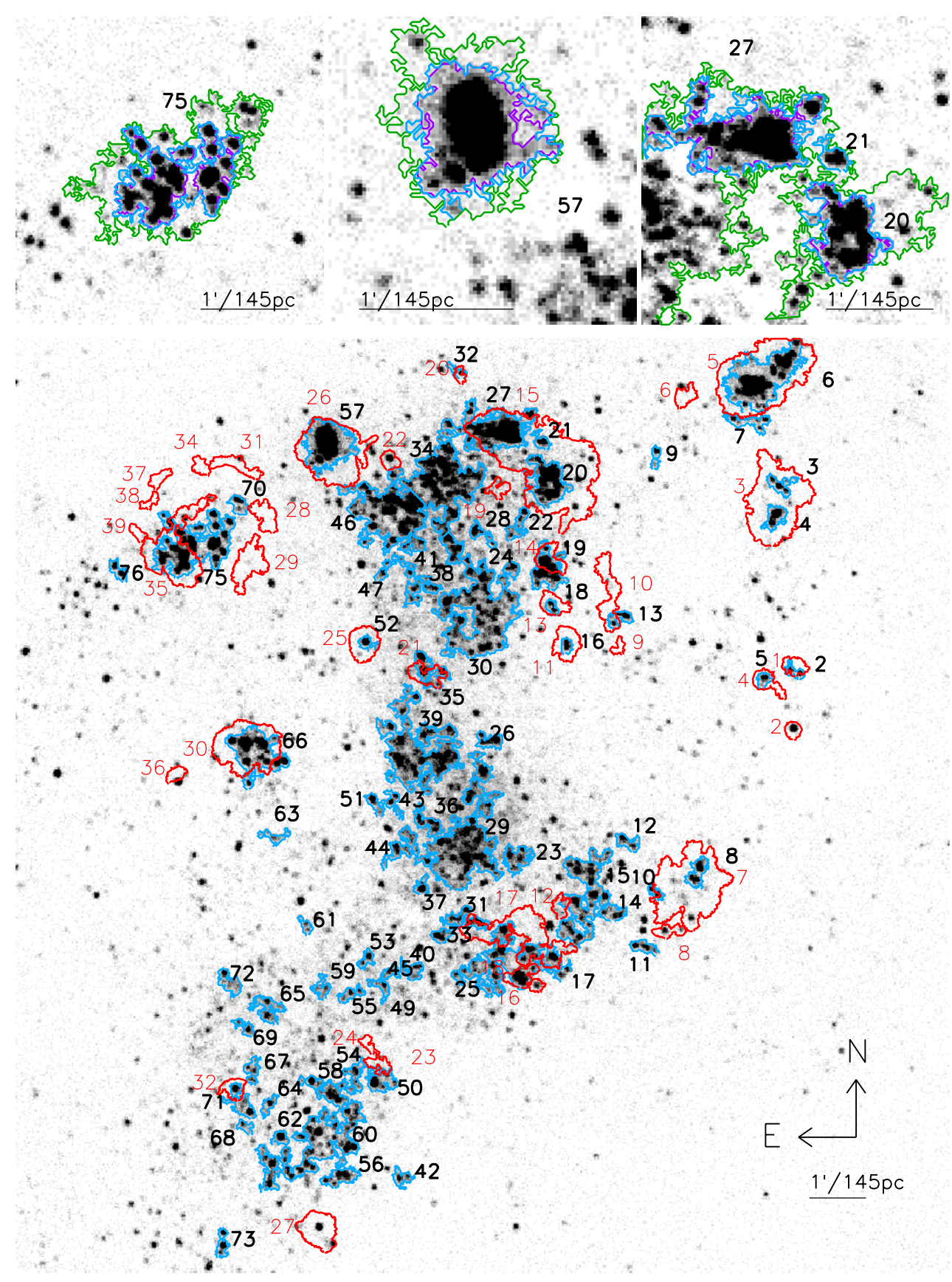

Figure 1. Contours of SF regions (blue) over the GALEX FUV image, defined with FUV surface brightness $>3 \sigma$ above the background level and area $>150$ arcsec ${ }^{2}$ $\left(860 \mathrm{pc}^{2}\right) . \mathrm{H} \alpha$ contours $(3 \sigma)$ are shown in red. Enlargements of regions 75, 57, 27, and 20 in the top panels illustrate the effect of the threshold choice for contour definition ( $1 \sigma$ in green, $3 \sigma$ in light blue, $4 \sigma$ in dark blue).

$\geqslant 5$ stars more massive than $10 \mathcal{M}_{\odot}$ i.e., earlier than spectral type B2V (with the parameters adopted by these authors). We will return to this point again later.

We choose to restrict the analysis sample with the area cut of $150 \operatorname{arcsec}^{2}$ after examining the distribution of stars with spectral type earlier than $\mathrm{B} 2 \mathrm{~V}^{8}$ inside the FUV-defined contours. In Figure 2, we plot the FUV magnitude versus the areas of the SF regions: those containing $\geqslant 5$ blue massive stars are shown with dots. A cut by an area of $\geqslant 150 \operatorname{arcsec}^{2}$ includes $94 \%$ of these regions in the sample, and very few regions containing less than five blue stars $(20 \%)$. We examined the alternative

\footnotetext{
8 Selected from the photometry of Massey et al. (2007b) to have $(B-V)_{0}<-0.15$ and $M_{V}<-2.5$, after the reddening correction is applied, as described in Section 3.1.
}

option of a brightness cut, which is often used in studies of more distant galaxies. Such criterion would either include fewer $\mathrm{SF}$ regions with five or more blue stars, or more regions with less than five blue stars, in the analysis sample. For example, a brightness cut at FUV $\leqslant 20.2$ mag includes in the analysis sample $94 \%$ of the regions with $\geqslant 5$ blue stars (the same fraction as our area cut of $\geqslant 150 \operatorname{arcsec}^{2}$ ), but $44 \%$ of the selected regions would contain less than five blue stars. Therefore, a cut by area better satisfies our requirement of a minimum number of blue stars within a source contour, including in the analysis sample as many as possible of the clusters having $\leqslant 5$ massive stars, and as few as possible clusters with $<5$ massive stars. A brightness or luminosity cut would also strongly be affected by extinction or extinction correction (see Section 3.2). We point out that 


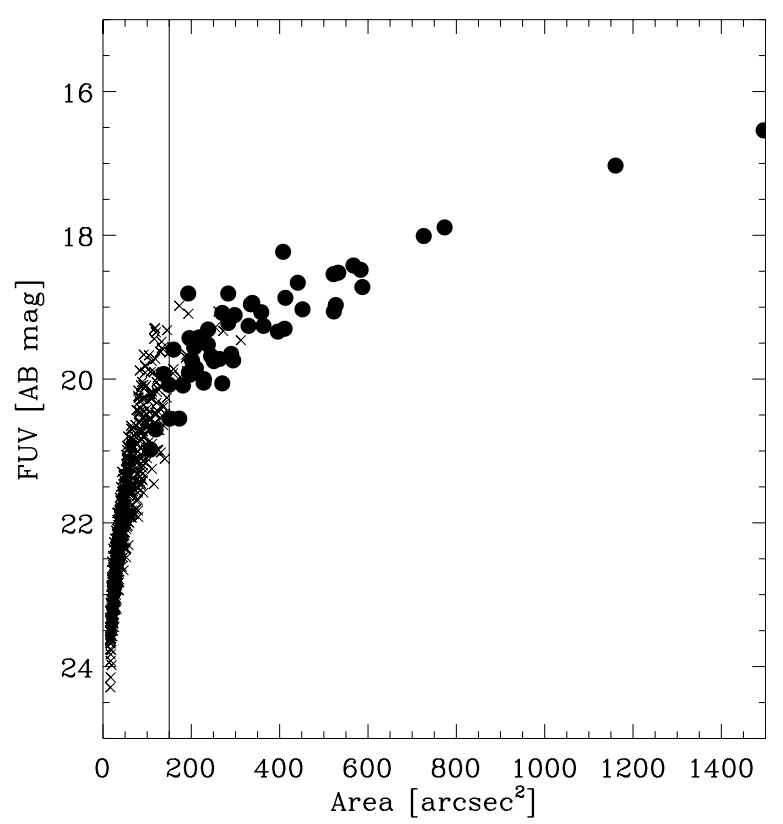

Figure 2. FUV magnitude vs. area of FUV-defined SF regions. Regions containing five or more blue stars (see the text) are marked with filled circles. A cut by area at $150 \operatorname{arcsec}^{2}$, adopted for our analysis sample, is shown with a vertical line. A sample cut by flux such as to retain most of the SF regions with $\geqslant 5$ hot stars would include in the analysis sample several sources with less than five stars.

this criterion may not necessarily be the best choice for more distant galaxies where similar data would give a lower spatial resolution, or for galaxies where star formation is less sparse. In the specific case of NGC 6822, such criterion, more precise than a luminosity cut for our purpose, could be tested and tuned given the vicinity of the galaxy and the detailed information on its stellar population. Finally, a cut by area may eliminate young compact clusters and may be undesirable in disk galaxies, for example, where young compact star clusters abound (e.g., Bianchi et al. 1999 and Chandar et al. 1999 for M33; Hodge et al. 2010 and Y.-B. Kang et al. 2011, in preparation for M31). In NGC 6822, there are very few such compact clusters and their exclusion would not change our results. This work aims to detect unbound OB associations and SF complexes, not compact star clusters. Another advantage of the area cut is that it effectively excludes foreground stars.

The resulting analysis sample includes 77 FUV-defined sources with area $\geqslant 150 \operatorname{arcsec}^{2}$ and brightness $\lesssim 26.8$ mag $\operatorname{arcsec}^{-2}$, within a 0.2 radius $(1.72 \mathrm{kpc})$ of the center of NGC 6822 . The 0.2 radius is 1.5 times the optical semimajor axis of the galaxy $\left(7^{\prime} .8\right.$ at $\left.\sim 25 \mathrm{mag} \operatorname{arcsec}^{-2}\right)$ given by Karachentsev et al. (2004). $\mathrm{H} \alpha$ emission is detected out to a radius of $R_{\mathrm{H} \alpha}=1.65 \mathrm{kpc}$ (Hunter \& Elmegreen 2004; see also Section 2.3), and the H I disk (de Blok \& Walter 2006) also exceeds the optical size of the galaxy (see also Bianchi 2011). Our sample extends to a slightly larger area than that of Melena et al. (2009). ${ }^{9}$

The areas of the selected SF regions range from 150 to $5400 \operatorname{arcsec}^{2}\left(860-3 \times 10^{4} \mathrm{pc}^{2}\right)$. Table 1 gives identification, coordinates of the "centroids" (the median $\alpha$ and $\delta$ values of the pixels included in the contours), and areas of the FUV-defined regions, ordered by increasing R.A. The contours are shown in blue in Figure 1 over the GALEX FUV image. In the next

\footnotetext{
9 The Melena et al. (2009) sample of SF regions is within $1.65 \mathrm{kpc}$, using the coordinates of their Table 2, in spite of their claim that it extends to $6 \mathrm{kpc}$.
}

section, we describe the photometry measurements, which are used in Section 3 to derive ages and masses.

\subsection{UV Photometry of the Star-forming Regions}

For photometric measurements, we used the intensity map ("int") images (in units of counts $\mathrm{s}^{-1}$ pixel $^{-1}$ ) generated by the GALEX pipeline dividing the count map by the relative response map. ${ }^{10}$ We measured the FUV and NUV flux of each SF region within its FUV-defined contour and the local background. The background was measured over an area defined by smoothing the source contour and expanding it by 3 pixels (inner background contour) and 9 pixels (outer contour), i.e., creating a 6 pixel wide "annulus" around the source, which follows its shape. The median of the flux pixel ${ }^{-1}$ in the background region, excluding portions of nearby sources falling in the background annulus, was then subtracted from every pixel inside the source contour. The conversion from (counts $\mathrm{s}^{-1}$ ) to magnitudes in the $\mathrm{AB}$ photometric system was performed using zero points, $\mathrm{ZP}=18.82 \mathrm{mag}$ (FUV) and $20.08 \mathrm{mag}$ (NUV; Morrissey et al. 2007). We calculated the photometric errors as $\Delta$ mag $\approx$ $2.5 / \ln (10) \times \frac{N}{S}=1.09 \frac{N}{S}$, where $S$ is the flux from the source in the aperture and $N$ is the quadratic sum of all the noises affecting the image. $S$ is expressed by $S=F \times$ EPADU, where $F$ is the flux in counts, and EPADU is the conversion factor from $\mathrm{ADU}$ to $e^{-}$, for GALEX EPADU $=1$. We consider the Poisson noise of the photon flux, and the background fluctuations to dominate, so we used the following expression to estimate the noise: $N=\sqrt{N_{\text {source }}^{2}+N_{\text {background }}^{2}+A \sigma^{2}}=\sqrt{S+S_{\text {background }}+A \sigma^{2}}$, where $A$ is the area of the source (in pixels) and $\sigma$ is the standard deviation among the pixels in the background annulus (in counts). The resulting FUV and NUV magnitudes and their errors are listed in Table 1.

The total flux from the selected SF regions is $45 \%$ of the integrated FUV flux from NGC $6822\left(F_{\text {FUVtot }}=1.05 \pm 0.07 \times\right.$ $10^{-9} \mathrm{erg} \mathrm{s}^{-1} \mathrm{~cm}^{-2}$ or $\left.\mathrm{FUV}_{\text {tot }}=12.1 \pm 0.07 \mathrm{mag} \mathrm{AB}\right)$ and $35 \%$ of the total NUV flux ( $F_{\text {NUVtot }}=1.09 \pm 0.04 \times$ $10^{-9} \mathrm{erg} \mathrm{s}^{-1} \mathrm{~cm}^{-2}$ or $\mathrm{NUV}_{\text {tot }}=11.7 \pm 0.04 \mathrm{mag} \mathrm{AB}$ ), measured from the pipeline sky-subtracted image in an aperture of 0.2 radius. The flux not included in our SF regions comes from smaller sources excluded by our area cut ( $10 \%$ of $\left.F_{\text {FUVtot }}\right)$, from older diffuse populations, and from scattered emission from SF regions. The fraction of flux included in the selected SF sites is lower in NUV than in FUV because foreground stars and diffuse light from older populations are more conspicuous at longer wavelengths.

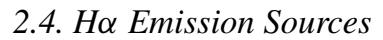

We also used the publicly available CTIO $\mathrm{H} \alpha$ image from the survey of Massey et al. (2007a) to define contours of $\mathrm{H} \alpha$ emitting regions. The $\mathrm{H} \alpha$ image has an exposure of $300 \mathrm{~s}$, a scale of 0.27 pixel $^{-1}$, and a resolution of $0^{\prime \prime} .9(2.2 \mathrm{pc}$ at the distance to NGC 6822). We used the $V$ and $R$ images from the same survey (Massey et al. 2007b) to correct the $\mathrm{H} \alpha$ image for continuum, by subtracting from it a linear combination of the $V$ and $R$ images, scaled to match the intensity of the continuum sources. We define contours of $\mathrm{H} \alpha$ emitting regions using a threshold of $3 \sigma$ above the background, corresponding to a surface brightness of $3 \times 10^{-17} \mathrm{erg} \mathrm{s}^{-1} \mathrm{~cm}^{-2} \operatorname{arcsec}^{-2}$. The $\mathrm{H} \alpha$-defined contours are drawn in red in Figure 1. They generally follow the $\mathrm{H} \alpha$ contours given by Hodge et al. (1988, 1989), who defined them in a similar way using a threshold of $2 \times 10^{-17} \mathrm{erg} \mathrm{s}^{-1} \mathrm{~cm}^{-2} \operatorname{arcsec}^{-2}$.

\footnotetext{
$\overline{10}$ http://galexgi.gsfc.nasa.gov/Documents/ERO_data_description_3.htm
} 
Table 1

FUV-selected SF Regions

\begin{tabular}{|c|c|c|c|c|c|c|c|c|c|}
\hline \multirow[t]{2}{*}{$\mathrm{ID}^{\mathrm{a}}$} & \multirow[t]{2}{*}{$\alpha(\mathrm{J} 2000)^{\mathrm{b}}$} & \multirow[t]{2}{*}{$\delta(\mathrm{J} 2000)^{\mathrm{b}}$} & \multirow{2}{*}{$\begin{array}{c}\text { Area } \\
\left(\operatorname{arcsec}^{2} / \mathrm{pc}^{2}\right) \\
\end{array}$} & \multirow{2}{*}{$\begin{array}{c}\text { FUV }^{\mathrm{c}} \\
\text { (AB mag) } \\
\end{array}$} & \multirow{2}{*}{$\begin{array}{c}\mathrm{NUV}^{\mathrm{c}} \\
\text { (AB mag) }\end{array}$} & \multicolumn{2}{|c|}{$\begin{array}{l}\text { Background } \\
\text { Fraction }^{\mathrm{d}}\end{array}$} & \multirow{2}{*}{$\begin{array}{c}E(B-V)^{\mathrm{e}} \\
(\mathrm{mag}) \text { and } 1 \sigma \text { scatter }\end{array}$} & \multirow[t]{2}{*}{ Comment $^{\mathrm{f}}$} \\
\hline & & & & & & FUV & NUV & & \\
\hline EB-FUV 1 & 194414.46 & -144639.1 & $190 / 1110$ & $18.81 \pm 0.02$ & $18.59 \pm 0.01$ & 0.08 & 0.13 & $0.50 \pm 0.12$ & \\
\hline EB-FUV $2^{\mathrm{g}}$ & 194431.00 & -144723.5 & $190 / 1090$ & $19.70 \pm 0.03$ & $19.73 \pm 0.02$ & 0.21 & 0.30 & $0.35 \pm 0.07$ & \\
\hline EB-FUV $3^{\mathrm{g}}$ & 194431.63 & -144404.0 & $340 / 1950$ & $18.94 \pm 0.02$ & $19.09 \pm 0.01$ & 0.19 & 0.31 & $0.35 \pm 0.17$ & Part of OB2 \\
\hline EB-FUV $4^{\mathrm{g}}$ & 194432.45 & -144445.2 & $410 / 2350$ & $18.23 \pm 0.01$ & $18.35 \pm 0.01$ & 0.15 & 0.25 & $0.36 \pm 0.07$ & Part of OB2 \\
\hline EB-FUV $5^{g}$ & 194433.02 & -144732.5 & $190 / 1090$ & $19.66 \pm 0.03$ & $19.70 \pm 0.02$ & 0.20 & 0.31 & $0.41 \pm 0.08$ & \\
\hline EB-FUV $6^{\mathrm{g}}$ & 194433.13 & -144201.7 & $3440 / 19810$ & $15.67 \pm 0.01$ & $15.57 \pm 0.01$ & 0.12 & 0.16 & $0.31 \pm 0.13$ & $\mathrm{OB} 1$ and $\mathrm{OB} 3$ \\
\hline EB-FUV 7 & 194433.95 & -144257.2 & $450 / 2600$ & $19.03 \pm 0.02$ & $19.03 \pm 0.02$ & 0.28 & 0.38 & $0.31 \pm 0.20$ & \\
\hline EB-FUV $8^{g}$ & 194437.82 & -145057.3 & $440 / 2540$ & $18.66 \pm 0.02$ & $18.78 \pm 0.01$ & 0.24 & 0.36 & $0.32 \pm 0.11$ & OB4 \\
\hline EB-FUV 9 & 194440.88 & -144340.0 & $160 / 910$ & $19.92 \pm 0.04$ & $20.16 \pm 0.05$ & 0.21 & 0.39 & $0.44 \pm 0.15$ & \\
\hline EB-FUV $10^{\mathrm{g}}$ & 194440.98 & -145119.8 & $160 / 900$ & $19.56 \pm 0.03$ & $19.57 \pm 0.02$ & 0.19 & 0.28 & $0.66 \pm 0.21$ & \\
\hline EB-FUV 11 & 194441.70 & -145217.5 & $300 / 1720$ & $19.11 \pm 0.02$ & $19.17 \pm 0.01$ & 0.20 & 0.31 & $0.36 \pm 0.11$ & \\
\hline EB-FUV 12 & 194442.74 & -145025.0 & $280 / 1630$ & $19.22 \pm 0.03$ & $18.97 \pm 0.01$ & 0.23 & 0.30 & $0.42 \pm 0.04$ & \\
\hline EB-FUV $13^{\mathrm{g}}$ & 194443.67 & -144630.3 & $280 / 1630$ & $18.81 \pm 0.02$ & $18.72 \pm 0.01$ & 0.15 & 0.24 & $0.38 \pm 0.12$ & \\
\hline EB-FUV 14 & 194443.82 & -145136.3 & $360 / 2090$ & $19.26 \pm 0.03$ & $19.31 \pm 0.02$ & 0.32 & 0.45 & $0.40 \pm 0.18$ & \\
\hline EB-FUV 15 & 194446.05 & -145128.1 & $2700 / 15550$ & $16.89 \pm 0.01$ & $16.78 \pm 0.01$ & 0.30 & 0.41 & $0.35 \pm 0.14$ & \\
\hline EB-FUV $16^{\mathrm{g}}$ & 194447.24 & -144659.6 & $160 / 920$ & $19.59 \pm 0.03$ & $19.46 \pm 0.02$ & 0.18 & 0.28 & $0.48 \pm 0.17$ & \\
\hline EB-FUV 17 & 194447.91 & -145240.8 & $190 / 1100$ & $19.89 \pm 0.04$ & $19.84 \pm 0.03$ & 0.28 & 0.38 & $0.40 \pm 0.05$ & \\
\hline EB-FUV $18^{\mathrm{g}}$ & 194448.43 & -144616.8 & $150 / 870$ & $19.63 \pm 0.03$ & $19.64 \pm 0.02$ & 0.19 & 0.32 & $0.29 \pm 0.00$ & \\
\hline EB-FUV $19^{g}$ & 194448.90 & -144530.3 & $1160 / 6680$ & $17.03 \pm 0.01$ & $16.93 \pm 0.01$ & 0.17 & 0.25 & $0.41 \pm 0.13$ & OB7 \\
\hline EB-FUV $20^{\mathrm{g}}$ & 194449.36 & -144400.3 & $1500 / 8620$ & $16.54 \pm 0.01$ & $16.59 \pm 0.01$ & 0.17 & 0.25 & $0.34 \pm 0.14$ & OB6 \\
\hline EB-FUV $21^{\mathrm{g}}$ & 194449.52 & -144315.3 & $200 / 1130$ & $19.43 \pm 0.03$ & $19.52 \pm 0.02$ & 0.28 & 0.38 & $0.30 \pm 0.15$ & \\
\hline EB-FUV 22 & 194450.96 & -144452.8 & $360 / 2060$ & $19.07 \pm 0.03$ & $19.18 \pm 0.02$ & 0.35 & 0.49 & $0.32 \pm 0.14$ & \\
\hline EB-FUV 23 & 194451.06 & -145043.1 & $590 / 3380$ & $18.72 \pm 0.03$ & $18.73 \pm 0.02$ & 0.38 & 0.52 & $0.45 \pm 0.15$ & \\
\hline EB-FUV 24 & 194451.53 & -144539.3 & $200 / 1160$ & $19.74 \pm 0.04$ & $19.82 \pm 0.03$ & 0.37 & 0.51 & $0.31 \pm 0.12$ & \\
\hline EB-FUV $25^{\mathrm{g}}$ & 194452.35 & -145238.6 & $3480 / 20030$ & $16.49 \pm 0.01$ & $16.42 \pm 0.01$ & 0.28 & 0.38 & $0.40 \pm 0.16$ & Part of OB5 \\
\hline EB-FUV 26 & 194453.13 & -144837.1 & $330 / 1930$ & $18.96 \pm 0.02$ & $18.97 \pm 0.02$ & 0.28 & 0.44 & $0.44 \pm 0.11$ & \\
\hline EB-FUV $27^{g}$ & 194453.39 & -144302.6 & $2760 / 15900$ & $15.67 \pm 0.01$ & $15.55 \pm 0.01$ & 0.14 & 0.18 & $0.41 \pm 0.15$ & OB8 \\
\hline EB-FUV 28 & 194453.44 & -144459.6 & $270 / 1560$ & $19.08 \pm 0.03$ & $19.17 \pm 0.02$ & 0.33 & 0.45 & $0.32 \pm 0.12$ & \\
\hline EB-FUV 29 & 194454.48 & -145016.1 & $5060 / 29160$ & $16.24 \pm 0.01$ & $16.12 \pm 0.01$ & 0.39 & 0.51 & $0.42 \pm 0.18$ & \\
\hline EB-FUV 30 & 194454.79 & -144616.8 & $5400 / 31100$ & $16.15 \pm 0.01$ & $16.00 \pm 0.01$ & 0.37 & 0.48 & $0.38 \pm 0.18$ & \\
\hline EB-FUV 31 & 194455.00 & -145149.1 & $520 / 3010$ & $18.54 \pm 0.02$ & $18.68 \pm 0.02$ & 0.30 & 0.46 & $0.44 \pm 0.15$ & \\
\hline EB-FUV 32 & 194455.77 & -144204.8 & $160 / 920$ & $19.86 \pm 0.03$ & $20.05 \pm 0.02$ & 0.23 & 0.35 & $0.22 \pm 0.02$ & \\
\hline EB-FUV 33 & 194456.29 & -145201.1 & $250 / 1460$ & $19.28 \pm 0.03$ & $19.13 \pm 0.02$ & 0.31 & 0.38 & $0.31 \pm 0.06$ & \\
\hline EB-FUV 34 & 194456.34 & -144405.6 & $3920 / 22560$ & $16.07 \pm 0.01$ & $15.99 \pm 0.01$ & 0.31 & 0.39 & $0.33 \pm 0.19$ & OB9 \\
\hline EB-FUV $35^{\mathrm{g}}$ & 194457.48 & -144728.8 & $730 / 4180$ & $18.01 \pm 0.02$ & $17.74 \pm 0.01$ & 0.30 & 0.39 & $0.41 \pm 0.18$ & \\
\hline EB-FUV 36 & 194457.89 & -145004.1 & $410 / 2380$ & $18.87 \pm 0.03$ & $18.74 \pm 0.02$ & 0.38 & 0.49 & $0.33 \pm 0.13$ & \\
\hline EB-FUV 37 & 194458.10 & -145111.6 & $210 / 1190$ & $19.56 \pm 0.03$ & $19.45 \pm 0.07$ & 0.34 & 0.45 & $0.23 \pm 0.02$ & \\
\hline EB-FUV 38 & 194458.26 & -144557.3 & $530 / 3040$ & $18.97 \pm 0.03$ & $19.09 \pm 0.03$ & 0.46 & 0.63 & $0.41 \pm 0.22$ & \\
\hline EB-FUV 39 & 194458.36 & -144841.6 & $4220 / 24320$ & $16.27 \pm 0.01$ & $16.05 \pm 0.01$ & 0.32 & 0.43 & $0.42 \pm 0.20$ & \\
\hline EB-FUV 40 & 194458.97 & -145241.6 & $290 / 1700$ & $19.74 \pm 0.04$ & $19.89 \pm 0.03$ & 0.43 & 0.56 & $0.32 \pm 0.13$ & \\
\hline EB-FUV 41 & 194459.29 & -144531.8 & $180 / 1040$ & $20.09 \pm 0.05$ & $20.06 \pm 0.04$ & 0.44 & 0.57 & $0.60 \pm 0.25$ & \\
\hline EB-FUV 42 & 194459.60 & -145622.1 & $240 / 1410$ & $19.68 \pm 0.03$ & $19.35 \pm 0.02$ & 0.23 & 0.28 & $0.38 \pm 0.09$ & \\
\hline EB-FUV 43 & 194500.07 & -144947.6 & $260 / 1520$ & $19.72 \pm 0.04$ & $19.87 \pm 0.03$ & 0.39 & 0.59 & $0.47 \pm 0.14$ & \\
\hline EB-FUV 44 & 194500.22 & -145038.6 & $330 / 1900$ & $19.26 \pm 0.03$ & $19.32 \pm 0.03$ & 0.33 & 0.48 & $0.32 \pm 0.05$ & \\
\hline EB-FUV 45 & 194500.58 & -145246.8 & $170 / 990$ & $20.55 \pm 0.06$ & $20.71 \pm 0.06$ & 0.52 & 0.64 & $0.41 \pm 0.08$ & \\
\hline EB-FUV 46 & 194500.73 & -144443.8 & $4170 / 24030$ & $16.09 \pm 0.01$ & $16.06 \pm 0.01$ & 0.29 & 0.40 & $0.30 \pm 0.19$ & OB11 \\
\hline EB-FUV 47 & 194500.73 & -144534.8 & $190 / 1120$ & $19.91 \pm 0.04$ & $19.98 \pm 0.04$ & 0.38 & 0.55 & $0.41 \pm 0.14$ & \\
\hline EB-FUV 48 & 194500.94 & -145853.6 & $200 / 1140$ & $19.40 \pm 0.02$ & $19.43 \pm 0.01$ & 0.13 & 0.22 & $0.26 \pm 0.04$ & \\
\hline EB-FUV 49 & 194501.05 & -145258.8 & $230 / 1320$ & $20.00 \pm 0.05$ & $19.97 \pm 0.03$ & 0.45 & 0.56 & $0.40 \pm 0.10$ & \\
\hline EB-FUV 50 & 194501.20 & -145433.3 & $570 / 3270$ & $18.42 \pm 0.02$ & $18.02 \pm 0.01$ & 0.26 & 0.28 & $0.38 \pm 0.12$ & \\
\hline EB-FUV 51 & 194501.41 & -144946.8 & $220 / 1250$ & $19.42 \pm 0.03$ & $19.32 \pm 0.02$ & 0.25 & 0.37 & $0.40 \pm 0.23$ & \\
\hline EB-FUV $52^{\mathrm{g}}$ & 194501.93 & -144655.8 & $170 / 990$ & $18.98 \pm 0.02$ & $18.73 \pm 0.01$ & 0.16 & 0.22 & $0.40 \pm 0.09$ & \\
\hline EB-FUV 53 & 194502.23 & -145233.3 & $170 / 1010$ & $19.97 \pm 0.04$ & $19.63 \pm 0.02$ & 0.35 & 0.38 & $0.38 \pm 0.07$ & \\
\hline EB-FUV 54 & 194503.02 & -145433.3 & $400 / 2280$ & $19.34 \pm 0.03$ & $19.25 \pm 0.02$ & 0.39 & 0.49 & $0.40 \pm 0.14$ & \\
\hline EB-FUV 55 & 194503.22 & -145307.1 & $250 / 1450$ & $19.75 \pm 0.04$ & $19.65 \pm 0.03$ & 0.39 & 0.48 & $0.33 \pm 0.13$ & \\
\hline EB-FUV 56 & 194504.05 & -145614.6 & $580 / 3360$ & $18.48 \pm 0.02$ & $18.33 \pm 0.01$ & 0.22 & 0.31 & $0.33 \pm 0.09$ & \\
\hline EB-FUV $57^{\mathrm{g}}$ & 194504.77 & -144325.8 & $2150 / 12400$ & $15.46 \pm 0.01$ & $15.48 \pm 0.01$ & 0.08 & 0.13 & $0.36 \pm 0.16$ & OB13 \\
\hline EB-FUV 58 & 194505.03 & -145445.3 & $770 / 4460$ & $17.89 \pm 0.01$ & $17.76 \pm 0.01$ & 0.25 & 0.31 & $0.32 \pm 0.11$ & \\
\hline EB-FUV 59 & 194505.44 & -145259.6 & $270 / 1560$ & $20.06 \pm 0.05$ & $20.00 \pm 0.04$ & 0.48 & 0.57 & $0.28 \pm 0.23$ & \\
\hline EB-FUV 60 & 194506.53 & -145552.8 & $3630 / 20880$ & $16.56 \pm 0.01$ & $16.53 \pm 0.01$ & 0.29 & 0.38 & $0.31 \pm 0.12$ & OB12 \\
\hline EB-FUV 61 & 194506.74 & -145155.1 & $150 / 870$ & $20.55 \pm 0.05$ & $20.36 \pm 0.24$ & 0.36 & 0.46 & $0.31 \pm 0.15$ & \\
\hline EB-FUV 62 & 194508.66 & -145541.6 & $190 / 1110$ & $19.09 \pm 0.03$ & $19.23 \pm 0.02$ & 0.20 & 0.31 & $0.27 \pm 0.02$ & \\
\hline EB-FUV 63 & 194508.91 & -145017.6 & $230 / 1310$ & $20.05 \pm 0.04$ & $19.97 \pm 0.03$ & 0.31 & 0.44 & $0.29 \pm 0.07$ & \\
\hline EB-FUV 64 & 194509.32 & -145504.1 & $210 / 1210$ & $19.85 \pm 0.04$ & $20.05 \pm 0.03$ & 0.33 & 0.48 & $0.40 \pm 0.27$ & \\
\hline
\end{tabular}




\begin{tabular}{|c|c|c|c|c|c|c|c|c|c|}
\hline \multirow{3}{*}{$\mathrm{ID}^{\mathrm{a}}$} & \multicolumn{7}{|c|}{$\begin{array}{c}\text { Table } 1 \\
\text { (Continued) }\end{array}$} & \multirow{3}{*}{$\begin{array}{c}E(B-V)^{\mathrm{e}} \\
(\mathrm{mag}) \text { and } 1 \sigma \text { scatter }\end{array}$} & \multirow{3}{*}{ Comment $^{\mathrm{f}}$} \\
\hline & $\alpha(\mathrm{J} 2000)^{\mathrm{b}}$ & $\delta(\mathrm{J} 2000)^{\mathrm{b}}$ & Area & $\mathrm{FUV}^{\mathrm{c}}$ & $\mathrm{NUV}^{\mathrm{c}}$ & \multicolumn{2}{|c|}{$\begin{array}{l}\text { Background } \\
\text { Fraction }^{\mathrm{d}}\end{array}$} & & \\
\hline & & & $\left(\operatorname{arcsec}^{2} / \mathrm{pc}^{2}\right)$ & (AB mag) & (AB mag) & FUV & NUV & & \\
\hline EB-FUV 65 & 194509.48 & -145323.5 & $520 / 3010$ & $19.06 \pm 0.03$ & $19.14 \pm 0.02$ & 0.39 & 0.51 & $0.33 \pm 0.09$ & \\
\hline EB-FUV 66 & 194510.31 & -144855.0 & $2100 / 12090$ & $16.49 \pm 0.01$ & $16.34 \pm 0.01$ & 0.17 & 0.23 & $0.26 \pm 0.16$ & OB14 \\
\hline EB-FUV 67 & 194510.31 & -145425.8 & $290 / 1670$ & $19.65 \pm 0.04$ & $19.82 \pm 0.03$ & 0.36 & 0.50 & $0.25 \pm 0.07$ & \\
\hline EB-FUV 68 & 194510.94 & -145526.5 & $150 / 870$ & $20.51 \pm 0.06$ & $20.89 \pm 0.06$ & 0.40 & 0.59 & $0.52 \pm 0.22$ & \\
\hline EB-FUV 69 & 194511.14 & -145340.0 & $200 / 1130$ & $19.94 \pm 0.04$ & $20.02 \pm 0.03$ & 0.33 & 0.46 & $0.41 \pm 0.15$ & \\
\hline EB-FUV 70 & 194511.33 & -144427.3 & $270 / 1570$ & $19.33 \pm 0.03$ & $19.44 \pm 0.08$ & 0.26 & 0.42 & $0.22 \pm 0.10$ & \\
\hline EB-FUV $71^{\mathrm{g}}$ & 194511.60 & -145458.8 & $530 / 3070$ & $18.52 \pm 0.02$ & $18.63 \pm 0.01$ & 0.27 & 0.39 & $0.35 \pm 0.14$ & \\
\hline EB-FUV 72 & 194512.17 & -145252.0 & $410 / 2370$ & $19.30 \pm 0.03$ & $19.37 \pm 0.02$ & 0.36 & 0.48 & $0.35 \pm 0.08$ & \\
\hline EB-FUV 73 & 194512.80 & -145734.0 & $260 / 1510$ & $19.06 \pm 0.02$ & $19.06 \pm 0.01$ & 0.16 & 0.24 & $0.26 \pm 0.04$ & \\
\hline EB-FUV 74 & 194513.47 & -145849.0 & $240 / 1370$ & $19.52 \pm 0.03$ & $19.42 \pm 0.02$ & 0.21 & 0.28 & $0.29 \pm 0.07$ & \\
\hline EB-FUV $75^{\mathrm{g}}$ & 194514.85 & -144501.8 & $3410 / 19630$ & $15.72 \pm 0.01$ & $15.86 \pm 0.01$ & 0.14 & 0.24 & $0.30 \pm 0.20$ & OB15 \\
\hline EB-FUV 76 & 194520.28 & -144537.0 & $240 / 1370$ & $19.31 \pm 0.03$ & $19.22 \pm 0.01$ & 0.21 & 0.29 & $0.28 \pm 0.21$ & \\
\hline EB-FUV 77 & 194530.58 & -145005.4 & $310 / 1800$ & $19.46 \pm 0.03$ & $19.72 \pm 0.02$ & 0.24 & 0.40 & $0.23 \pm 0.25$ & \\
\hline
\end{tabular}

\section{Notes.}

a Numbers correspond to the blue labels in Figure 1.

${ }^{b}$ Coordinates of the centroids of the contours (see Section 2.2).

${ }^{c}$ Photometric errors are estimated as explained in the text, without adding the uncertainties of the GALEX calibration zero points.

${ }^{\mathrm{d}}$ Fraction of background flux over total flux in the aperture.

${ }^{\mathrm{e}}$ The median of values derived for the massive stars (earlier than $B 2 V$ ) in each SF region (see Section 3.2).

${ }^{f}$ Names of OB associations after Hodge (1977).

$\mathrm{g}$ The FUV source is associated with $\mathrm{H} \alpha$ emission.

We used the calibration factor of 1 count $\mathrm{s}^{-1}=1.8 \times$ $10^{-16} \mathrm{erg} \mathrm{s}^{-1} \mathrm{~cm}^{-2}$ given by Massey et al. (2007a) for emissionline sources. We did not attempt to correct for [N II] emission line contamination, which we expect to not exceed a few percent of the flux. The average $F_{[\mathrm{N} \text { II] } 26584} / F_{\mathrm{H} \alpha}$ in the $\mathrm{H}_{\text {II }}$ regions measured by Pagel et al. (1980) is about 6\%; adopting $F_{[\mathrm{NII}] \lambda 6548} / F_{[\mathrm{N} \mathrm{II}] \lambda 6584} \approx 1 / 3$, we derive $F_{[\mathrm{N} \mathrm{II}]}(\lambda 6548+\lambda 6584) / F_{\mathrm{H} \alpha}$ $\approx 8 \%$. Since both $\left[\mathrm{N}_{\mathrm{II}}\right] \lambda \lambda 6548,6584$ fall in the wings of the $50 \AA$ wide filter passband centered at $\mathrm{H} \alpha$, we expect the actual contribution from [N II] emission to be of the order of $2 \%-3 \%$.

The $\mathrm{H} \alpha$ measurements are listed in Table 2. In the last column, we give the cross-identifications with $\mathrm{H} \alpha$ sources previously defined by Hubble (1925; $\mathrm{H}$ followed by a roman number), Hodge (1977; Ho followed by an arabic number), Kinman et al. (1979; K followed by a Greek letter), Killen \& Dufour (1982; KD followed by an arabic number), and Hodge et al. (1988; HK followed by an arabic number). For regions with $\mathrm{H} \alpha$ surface brightness $>1 \times 10^{-16} \mathrm{erg} \mathrm{s}^{-1} \mathrm{~cm}^{-2} \operatorname{arcsec}^{-2}$, our measurements agree within $\sim 25 \%$ with those by Hodge et al. (1989), after de-correcting the latter for extinction with $A_{\mathrm{H} \alpha}=0.9$ (O'Dell et al. 1999). The total $\mathrm{H} \alpha$ flux from the $\mathrm{H} \alpha$ emitting regions, $F_{\mathrm{H} \alpha}=1.8 \pm 0.2 \times 10^{-11} \mathrm{erg} \mathrm{s}^{-1} \mathrm{~cm}^{-2}$ (the sum of all measurements in Table 2), is higher by about $2 \%$ than the total flux from Table 1 of Hodge et al. (1989), most of the difference coming from faint $\mathrm{H}$ II regions not covered by the imaging of Hodge et al. (1988). We further checked our $\mathrm{H} \alpha$ calibration and continuum-source subtraction by measuring the flux from the $\mathrm{H}$ II regions Hubble $\mathrm{V}$ and Hubble $\mathrm{X}$ surrounding OB8 and OB13 in $42^{\prime \prime} \times 42^{\prime \prime}$ square apertures, similar to the ones used by O'Dell et al. (1999): our measurements agree within 10\% with the values given by these authors.

\section{ANALYSIS}

The UV color-magnitude diagram of the FUV-selected SF regions is shown in Figure 3: in the left panel we plotted the
FUV surface brightness (mag $\operatorname{arcsec}^{-2}$ ), and in the right panel the total FUV magnitude. In the right panel, we also show FUV synthetic magnitudes for single-burst (coeval) populations (Bianchi 2011) at various ages, scaled for total stellar masses of $1 \times 10^{4} / 1 \times 10^{3} / 1 \times 10^{2} \mathcal{M}_{\odot}$ (solid/dashed/dash-dotted lines) . Models are reddened with $E(B-V)=0.22 \mathrm{mag}$ (the assumed foreground extinction) using MW-type extinction (green lines) and with an additional $E(B-V)=0.15$ using the extinction curve derived for stars in the LMC2 SF region by Misselt et al. (1999; blue lines). For SF regions located in the main body of the galaxy, the local background (which includes the diffuse older populations, more conspicuous in the NUV band) is significant, and therefore there is always a concern that its subtraction may lead to greater uncertainty than the formal errors indicate. An error in the FUV-NUV color would propagate to an error in the derived age and consequently to the derived mass. To verify that no bias is introduced by high background subtractions, we plotted with empty diamonds the sources for which the background amounts to more than $30 \%$ of the flux in the source contour. These high-background sources are distributed in color-magnitude space not differently from the other sources, confirming that no biases have been introduced by the critical background estimate procedure.

In the following sections, we estimate ages and masses of the SF regions by comparing their UV photometry with simple (single-burst) stellar population (SSP) model colors of different metallicities (e.g., Bianchi 2007, 2009, 2011), after the $E(B-V)$ is estimated for each SF region from resolved stellar photometry. The models are progressively reddened with various types of interstellar dust extinction.

\subsection{Interstellar Reddening}

Flux at UV wavelengths is very sensitive to extinction by interstellar dust, and in order to derive the age and mass of the $\mathrm{SF}$ regions from model analysis, we first estimated the amount of reddening in each. 
Table 2

$\mathrm{H} \alpha$ Emission Regions

\begin{tabular}{|c|c|c|c|c|c|}
\hline ID & $\alpha(\mathrm{J} 2000)^{\mathrm{a}}$ & $\delta(\mathrm{J} 2000)^{\mathrm{a}}$ & $\begin{array}{c}\text { Area } \\
\left(\operatorname{arcsec}^{2} / \mathrm{pc}^{2}\right)\end{array}$ & $\begin{array}{c}\text { H } \alpha \text { Flux } \\
\left(10^{-13} \mathrm{erg} \mathrm{sec}^{-1} \mathrm{~cm}^{-2}\right)\end{array}$ & Comment $^{b}$ \\
\hline EB-Ha 1 & 194430.89 & -144719.7 & $410 / 2380$ & $0.24 \pm 0.03$ & HK1 \\
\hline $\mathrm{EB}-\mathrm{H} \alpha 2$ & 194430.93 & -144829.8 & $250 / 1420$ & $1.77 \pm 0.11$ & $\mathrm{~K} \alpha, \mathrm{KD} 2 \mathrm{e}$ \\
\hline $\mathrm{EB}-\mathrm{H} \alpha 3$ & 194432.29 & -144414.7 & $4420 / 25440$ & $7.92 \pm 0.19$ & HII, Ho1, Ho3, HK2, HK4D, KD2, KD3 \\
\hline $\mathrm{EB}-\mathrm{H} \alpha 4$ & 194432.33 & -144740.9 & $540 / 3090$ & $1.22 \pm 0.18$ & $\mathrm{~K} \beta, \mathrm{KD} 5 \mathrm{e}$ \\
\hline $\mathrm{EB}-\mathrm{H} \alpha 5$ & 194432.90 & -144202.6 & $5990 / 34510$ & $42.01 \pm 0.36$ & HI, HIII, Ho2,KD1, Ho4,KD4 \\
\hline $\mathrm{EB}-\mathrm{H} \alpha 6$ & 194438.72 & -144232.7 & $380 / 2220$ & $0.21 \pm 0.22$ & HK5D \\
\hline $\mathrm{EB}-\mathrm{H} \alpha 7$ & 194438.75 & -145110.2 & $4850 / 27940$ & $6.94 \pm 1.57$ & Ho5, KD8, KD9, HK6, KD7 \\
\hline $\mathrm{EB}-\mathrm{H} \alpha 8$ & 194439.98 & -145200.3 & $340 / 1950$ & $0.17 \pm 0.03$ & HK7, HK8 \\
\hline $\mathrm{EB}-\mathrm{H} \alpha 9$ & 194443.68 & -144700.2 & $160 / 930$ & $0.03 \pm 0.02$ & \\
\hline $\mathrm{EB}-\mathrm{H} \alpha 10$ & 194444.36 & -144557.9 & $1160 / 6710$ & $0.62 \pm 0.46$ & HK11D, HK12 \\
\hline $\mathrm{EB}-\mathrm{H} \alpha 11$ & 194447.46 & -144657.2 & $810 / 4660$ & $0.61 \pm 0.08$ & HK23 \\
\hline $\mathrm{EB}-\mathrm{H} \alpha 12$ & 194447.92 & -145129.5 & $320 / 1830$ & $0.22 \pm 0.05$ & \\
\hline $\mathrm{EB}-\mathrm{H} \alpha 13$ & 194448.44 & -144612.4 & $540 / 3110$ & $0.75 \pm 0.06$ & Ho7, KD11 \\
\hline $\mathrm{EB}-\mathrm{H} \alpha 14$ & 194448.45 & -144524.9 & $670 / 3840$ & $0.43 \pm 0.38$ & HK22, HK27, НK34 \\
\hline $\mathrm{EB}-\mathrm{H} \alpha 15$ & 194449.34 & -144348.0 & $9500 / 54710$ & $50.74 \pm 1.14$ & $\begin{array}{l}\text { HV, Ho6, KD12, KD21, HK13, HK15, HK16, HK17, HK19, } \\
\text { HK20, HK21, HK32, HK33, HK35, HK36, HK40, HK42, } \\
\text { HK44, HK53, Ho9, Ho11, KD19, KD10 }\end{array}$ \\
\hline $\mathrm{EB}-\mathrm{H} \alpha 16$ & 194449.73 & -145257.8 & $170 / 970$ & $1.05 \pm 0.05$ & $\mathrm{KD} 13, \mathrm{KD} 13 \mathrm{e}$ \\
\hline $\mathrm{EB}-\mathrm{H} \alpha 17$ & 194450.52 & -145206.9 & $3210 / 18520$ & $5.41 \pm 0.34$ & Ho10, K $\gamma, \mathrm{KD} 18, \mathrm{KD} 11 \mathrm{e}$ \\
\hline $\mathrm{EB}-\mathrm{H} \alpha 18$ & 194450.57 & -145245.9 & $550 / 3150$ & $2.81 \pm 0.27$ & Ho8, KD14, KD15, HK48, KD16, KD17 \\
\hline $\mathrm{EB}-\mathrm{H} \alpha 19$ & 194452.54 & -144411.0 & $300 / 1750$ & $0.16 \pm 0.04$ & HK55D \\
\hline $\mathrm{EB}-\mathrm{H} \alpha 20$ & 194455.23 & -144209.5 & $150 / 880$ & $0.08 \pm 0.02$ & \\
\hline $\mathrm{EB}-\mathrm{H} \alpha 21$ & 194457.66 & -144725.9 & $630 / 3620$ & $0.83 \pm 0.16$ & KD24, HK66, HK67 \\
\hline $\mathrm{EB}-\mathrm{H} \alpha 22$ & 194500.34 & -144340.1 & $300 / 1700$ & $0.24 \pm 0.25$ & HK73 \\
\hline $\mathrm{EB}-\mathrm{H} \alpha 23$ & 194501.23 & -145420.8 & $320 / 1840$ & $0.08 \pm 0.04$ & \\
\hline $\mathrm{EB}-\mathrm{H} \alpha 24$ & 194501.98 & -145400.2 & $190 / 1090$ & $0.07 \pm 0.02$ & \\
\hline $\mathrm{EB}-\mathrm{H} \alpha 25$ & 194502.38 & -144655.9 & $970 / 5570$ & $1.32 \pm 0.08$ & \\
\hline $\mathrm{EB}-\mathrm{H} \alpha 26$ & 194503.88 & -144335.3 & $3980 / 22910$ & $37.68 \pm 3.49$ & HX, HK77, HK78, HK79, HK80, Ho14, KD26 \\
\hline $\mathrm{EB}-\mathrm{H} \alpha 27$ & 194505.54 & -145720.6 & $1270 / 7330$ & $1.50 \pm 0.07$ & KD27, KD28 \\
\hline $\mathrm{EB}-\mathrm{H} \alpha 28$ & 194509.73 & -144439.8 & $640 / 3710$ & $0.27 \pm 0.05$ & HK97D \\
\hline $\mathrm{EB}-\mathrm{H} \alpha 29$ & 194510.91 & -144535.4 & $1210 / 6960$ & $0.52 \pm 0.19$ & HK98D \\
\hline $\mathrm{EB}-\mathrm{H} \alpha 30$ & 194510.94 & -144853.4 & $3130 / 18000$ & $3.74 \pm 0.28$ & Ho15, KD30 \\
\hline $\mathrm{EB}-\mathrm{H} \alpha 31$ & 194511.41 & -144347.7 & $580 / 3340$ & $0.35 \pm 0.18$ & \\
\hline $\mathrm{EB}-\mathrm{H} \alpha 32$ & 194511.95 & -145447.7 & $440 / 2540$ & $0.16 \pm 0.11$ & \\
\hline $\mathrm{EB}-\mathrm{H} \alpha 33$ & 194514.07 & -145850.5 & $160 / 910$ & $0.18 \pm 0.02$ & \\
\hline $\mathrm{EB}-\mathrm{H} \alpha 34$ & 194514.27 & -144347.6 & $260 / 1510$ & $0.09 \pm 0.10$ & \\
\hline $\mathrm{EB}-\mathrm{H} \alpha 35$ & 194515.83 & -144458.4 & $3050 / 17560$ & $3.24 \pm 0.20$ & Ho16, HK105D, HK106, HK107, KD31 \\
\hline $\mathrm{EB}-\mathrm{H} \alpha 36$ & 194516.03 & -144913.5 & $260 / 1470$ & $0.13 \pm 0.03$ & \\
\hline $\mathrm{EB}-\mathrm{H} \alpha 37$ & 194517.40 & -144401.5 & $260 / 1500$ & $0.09 \pm 0.06$ & \\
\hline $\mathrm{EB}-\mathrm{H} \alpha 38$ & 194517.89 & -144424.8 & $180 / 1040$ & $0.04 \pm 0.02$ & \\
\hline $\mathrm{EB}-\mathrm{H} \alpha 39$ & 194518.87 & -144457.0 & $190 / 1100$ & $0.09 \pm 0.19$ & HK108 \\
\hline
\end{tabular}

Notes.

a Coordinates of the "centroids" of the H $\alpha$ contours (see Section 2.2).

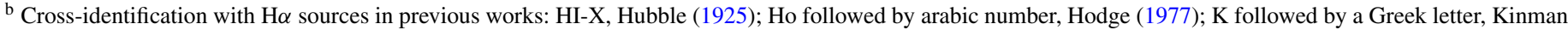
et al. (1979); KD followed by arabic number, Killen \& Dufour (1982); HK followed by arabic number, Hodge et al. (1988).

We used information from resolved stellar photometry, and estimated the $E(B-V)$ of the hot massive stars (selected with $(B-V)_{0}<-0.2$ and $M_{V}<-2.5$, i.e., earlier than about $\sim B 2 V)$ within each contour. For several OB associations, $E(B-V)$ values are available for individual stars, derived by Bianchi et al. (2001) and Bianchi \& Efremova (2006) from HST multi-band photometry (from UV to optical). For the most massive stars in six OB associations, we also have VLT spectroscopy (B. V. Efremova et al. 2011, in preparation), which confirms the results from $H S T$ photometry. For the regions without $H S T$ photometry, we derived $E(B-V)$ using the CTIO $U, B, V$ photometry of Massey et al. (2007b), with the standard "Q-method" (e.g., Bianchi \& Efremova 2006; Kang et al. 2009), and by comparing the observed $(U-B)$, $(B-V)$ colors with progressively reddened stellar model colors.
We accounted for extinction in each SF region using the median $E(B-V)$ value of the massive stars it contains. The values range from $E(B-V)=0.22$ (purely foreground extinction) to $0.66 \mathrm{mag}$, with a mean of $E(B-V)=0.36 \mathrm{mag}$, and are given in Table 1 . The mean $E(B-V)$ values are similarly distributed, ranging from $E(B-V)=0.21$ to $0.51 \mathrm{mag}$, with an average of $E(B-V)=0.37 \mathrm{mag}$. The typical $1 \sigma$ scatter (also given in Table 1) around the mean $E(B-V)$ in individual SF regions is $0.13 \mathrm{mag}$. The wide range of extinction values in the SF regions (not uncommon in SF galaxies) underscores the importance of accurate extinction corrections, particularly relevant in the UV regime, for deriving ages and masses. Other works adopt a generic assumption, for example, Melena et al. (2009) used a constant extinction of 0.27 mag for all their sample regions in NGC 6922, corresponding to $E(B-V)=0.05 \mathrm{mag}$ of internal extinction in addition to the $E(B-V)=0.22 \mathrm{mag}$ 

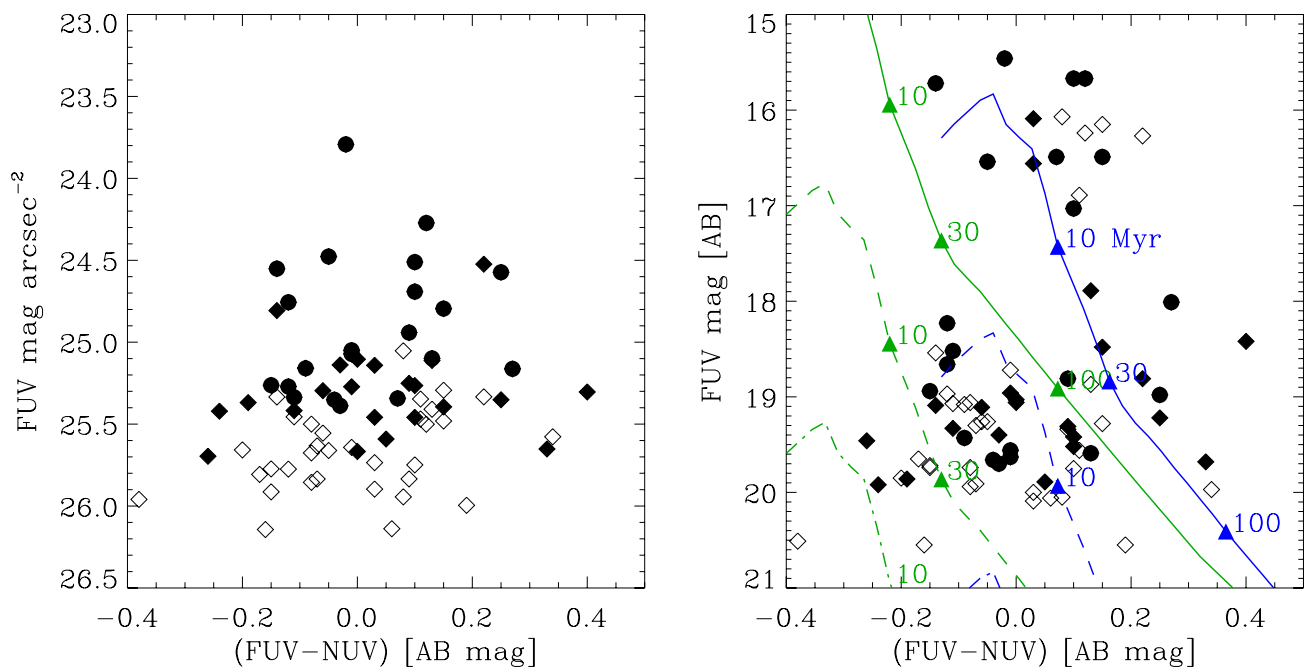

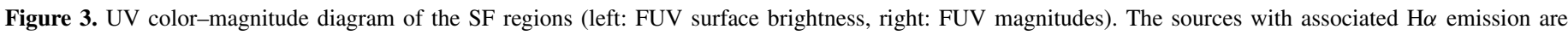

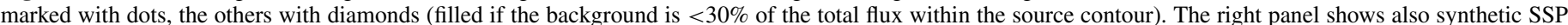

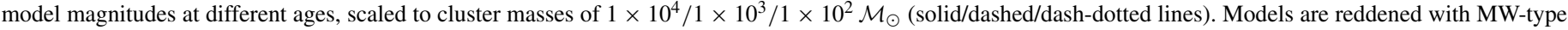

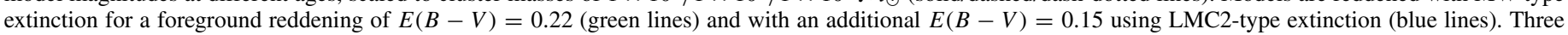
age values $(10,30$, and $100 \mathrm{Myr})$ are marked with triangles and labeled.

foreground reddening. Our results derived for individual regions (Table 1) indicate that a higher value is more typical. The model magnitudes plotted in Figure 3 illustrate how such assumptions affect the derived ages and masses; more model plots showing the effects of extinction can be found in Bianchi (2011).

We assume a foreground extinction of $E(B-V)=$ $0.22 \mathrm{mag}$, consistent with the minimum $E(B-V)$ estimated in this work, and with previous estimates by Bianchi et al. (2001), Bianchi \& Efremova (2006), and Massey et al. (2007b). Any additional extinction is considered to originate within NGC 6822.

While the derived $E(B-V)$ values are mostly based on optical photometry of the stars, and do not depend significantly on the type of dust, the selective extinction $A_{\lambda} / E(B-V)$ at UV wavelengths is known to strongly vary with environment. Therefore, the correction of UV magnitudes, and the resulting ages and masses, strongly depend on the adopted extinction curve (e.g., Bianchi 2011). In the analysis that follows, we consider four different types of internal extinction, found in the MW and in known low-metallicity SF environments: (1) MW-type extinction with $R_{V}=3.1$. In this case, the GALEX (FUV-NUV) color is basically reddening free (Bianchi 2011, and references therein); (2) the average extinction curve derived by Misselt et al. (1999) for LMC stars outside the 30 Doradus region (from here on, "LMC"), which gives an average color excess ratio for hot stars $\left(T_{\text {eff }}>10,000 \mathrm{~K}\right)$ of $E(\mathrm{FUV}-$ $\mathrm{NUV}) / E(B-V) \approx 1$; (3) the extinction curve in the LMC 30 Doradus region (from here on "LMC2") derived by Misselt et al. (1999), yielding $E(\mathrm{FUV}-\mathrm{NUV}) / E(B-V) \approx 2$; and (4) the extremely UV-steep extinction curve derived by Gordon \& Clayton (1998) for SMC stars (from here on, "SMC"), which yields $E(\mathrm{FUV}-\mathrm{NUV}) / E(B-V) \approx 5$.

\subsection{Ages and Masses of Star-forming Regions}

We derive the ages of the SF regions from their integrated FUV-NUV colors compared with the SSP models for lowmetallicity populations (see below), and masses from the age and UV magnitudes, accounting for reddening. We compared results obtained by adopting the different extinction curves mentioned in the previous section with information from resolved stellar photometry and $\mathrm{H} \alpha$, in order to assess what type of selective extinction is more appropriate.

We found that a uniform extinction type is not adequate for the whole sample of SF regions in NGC 6822. If we assume "average MW" extinction with $R_{V}=3.1$ for the whole sample (as adopted, e.g., by Hunter et al. 2010), the measured colors imply ages too old for several regions which show $\mathrm{H} \alpha$ emission and which appear to be a few Myr old in H-R diagrams from HST photometry (Bianchi et al. 2001). On the other hand, if we use a UV-steep extinction curve, "LMC2" for example, to deredden all SF regions, the (FUV-NUV) color for part of the sample is overcorrected, such that it appears unrealistic when compared with model predictions at any age. We found that different extinction curves are needed to bring the ages from GALEX integrated measurements in agreement with results from resolved studies for a subsample of well-studied regions. Regions 27, 57, 75, 19, 20 (approximately corresponding to OB8, OB13, OB15, OB7, and OB6 as defined by Hodge 1977), and 52, are included in the $H S T$ photometric studies by Bianchi et al. (2001) and Bianchi \& Efremova (2006); VLT spectroscopy of the most massive stars confirms the ages derived from HST photometry. By comparing results from integrated measurements with resolved stellar photometry of these wellstudied SF regions (outside the central part of the galaxy, where measurements are not complicated by significant diffuse older populations), and with information from $\mathrm{H} \alpha$ emission, we derived a criterion for choosing the type of extinction curve, and apply it to the rest of the sample. Specifically, the age information for the best-studied SF regions, with spectroscopy available for the hottest stars, was based on the presence (or absence) of O-type stars, W-R type stars, or B supergiants, as well as on the photometric H-R diagram of their stellar population, and we ruled out extinction curves giving very discrepant results from the GALEX color. For the FUV-bright regions clearly associated with $\mathrm{H} \alpha$ emission, we ruled out extinction curves yielding ages significantly older than $10 \mathrm{Myr}$ from integrated UV photometry. Finally, UV-steep extinction curves were excluded in the cases where they would yield an intrinsic FUV-NUV color bluer than any stellar population 


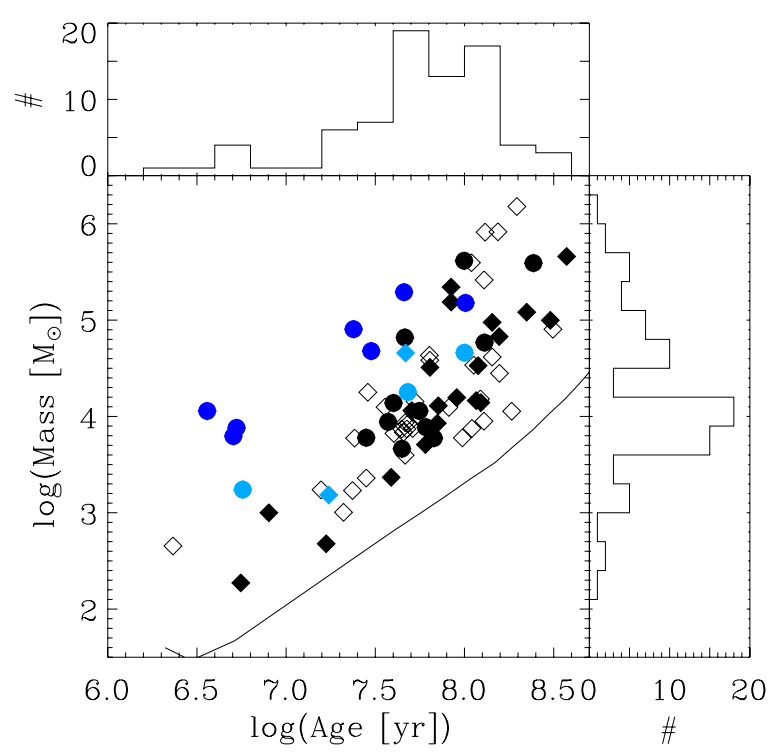

Figure 4. Masses vs. ages of the FUV-defined SF regions, derived from integrated UV magnitudes using SSP models with metallicity $Z=0.004$. We accounted for interstellar extinction using $E(B-V)$ values estimated for each $\mathrm{SF}$ region, and assuming a foreground component with $E(B-V)=0.22$ and MW-type dust $\left(R_{V}=3.1\right)$, and different extinction curves for the additional internal extinction as described in the text (black if "MW, $R_{V}=3.1$," light blue if "LMC," and dark blue if "LMC2"). As in the previous figure, UV sources associated with $\mathrm{H} \alpha$ emission are plotted with dots, and the rest with diamonds (empty symbols if the background is $>30 \%$ of the source flux). The black line shows the detection limit for a source with our minimum area and only foreground reddening.

model at any age. While derivation of the actual extinction curve would require UV spectroscopy of several stars in each region, and it is not possible with broadband photometry, the representative known curves examined give sufficiently different results (Table 3 ) that some of these assumptions could definitely be excluded in many cases. Some consistent trends within the subsample of SF regions with information on their stellar content allowed us to define general criteria.

For the youngest SF regions, a UV-steep extinction curve brings ages from integrated measurements into agreement with resolved $\mathrm{H}-\mathrm{R}$ diagram results. These regions are associated with strong $\mathrm{H} \alpha$ emission (plotted with circles in Figures 3 and 4) and typically have high FUV surface brightness, i.e., the SF is intense and the complex is very compact. Therefore, we assumed UV-steep extinction curves to correct for internal extinction of regions with surface brightness higher than 25.0 mag $\operatorname{arcsec}^{-2}$, and MW-type extinction for the rest of the sample. Among the high surface brightness SF regions, we found "LMC2"-type extinction to be preferable for sources with FUV $<17.5 \mathrm{mag}$, and the less steep "LMC" extinction to be better for sources fainter (in FUV) than this limit.

We stress that these criteria were derived ad hoc, to bring the results from integrated measurements into agreement with detailed studies of a subsample. HST photometry of sample regions for a wider sample of Local Group galaxies will be used to expand this comparison (Bianchi et al. 2010, 2011). However, the results are not surprising: for example, in a study of SF sites in M51, Calzetti et al. (2005) found starburst-like extinction to be applicable only to sites with the strongest star formation.

Two metallicity values, $Z=0.004$ and $Z=0.008$, were explored, encompassing the estimated metallicity of young stars in NGC 6822, $Z=0.006$ (Muschielok et al. 1999; Venn et al. 2001). Models with metallicity $Z=0.008$ yield slightly younger ages and consequently lower masses of the SF regions. The effect of metallicity on the derived age and mass varies with age and extinction type, as discussed by Kang et al. (2009; see their Figure 12), Bianchi (2009; in particular Figure 9), and Bianchi (2011).

Ages and masses of the SF regions, derived from integrated FUV, NUV photometry using models with metallicity $Z=$ 0.004 , and assuming three different extinction curves for internal extinction, are given in Table 3; the last column indicates which values are adopted in our analysis. These values are plotted in Figure 4. The gap in the age distribution at $10 \mathrm{Myr}$ is due to a slight degeneracy of the UV color in the range 6-11 Myr, due to RSGs emission (Fall et al. 2009). The uncertainty in the derived ages caused by this effect is smaller than the uncertainty introduced by the scatter in $E(B-V)$, and it does not affect our overall results significantly. We stress that the masses of the individual SF regions should not be interpreted in terms of the cluster mass function for two reasons. First, we defined irregular, mostly unbound, complexes. Second, we used a constant threshold throughout the galaxy, to derive source contours, in the interest of adhering to an objective criterion and a consistent flux limit. This choice inevitably may cause some sparse regions to break into subcomponents (but their ages, and the masses of each, would not be affected), and more diffuse regions to merge.

The magnitude limit of our sample, from the $3 \sigma$ detection threshold of $26.8 \mathrm{mag} \operatorname{arcsec}^{-2}$ and the area cut of $\geqslant 150 \operatorname{arcsec}^{2}$, translates (using the SSP models) into a mass detection limit increasing with age, shown with a black line in Figure 4 for a foreground reddening $E(B-V)=0.22 \mathrm{mag}$. The actual mass limit of the sample is higher because most sources have a higher reddening. As can be expected, the detection limit causes incompleteness for low masses at old ages.

For comparison, we also estimated the masses of the FUVselected SF regions from resolved stellar photometry. The mass of each SF region was derived by extrapolating the number of stars above $10 \mathcal{M}_{\odot}$ (corresponding to about $B 2 \mathrm{~V}$, and chosen with $M_{V}<-2.5$ and $\left.(B-V)_{0}<-0.2\right)$ and up to the most massive star still on the MS, to the interval $0.1-100 \mathcal{M}_{\odot}$. We assumed an IMF with $\alpha=2.3$ in the range $0.5 \mathcal{M}_{\odot}<M<$ $80 \mathcal{M}_{\odot}$, and $\alpha=1.3$ for $0.1 \mathcal{M}_{\odot}<M<0.5 \mathcal{M}_{\odot}$ after Kroupa (2001). The masses derived from the H-R diagrams agree with those from integrated UV photometry for SF regions younger than 10 Myr. For older regions, the H-R diagrams give lower masses than the integrated measurements, by up to a factor of 20 , probably because the most massive stars have evolved. The most discrepant regions have large areas and are in the main body of the galaxy, where the background is higher; they may be the result of merging of nearby regions expanding with age.

The possible contribution by foreground MW red dwarfs to the integrated GALEX flux of the SF regions was also estimated, since the density of foreground stars is significant in the direction of NGC 6822. We used our stellar model grids to estimate the possible contribution to the FUV and NUV fluxes from foreground stars of intermediate colors $\left(0.1<(B-V)_{0}<1.2\right.$ and $M_{V}<-2$; see, for example, Figure 6 of Bianchi \& Efremova 2006). The derived potential effect on the FUV-NUV color is very small ( -0.014 mag on average) and does not affect the results.

The main concerns to be addressed when deriving ages and masses of SF complexes by comparison with SSP model colors are: (1) the degree of coevality of the stellar complex and applicability of the SSP assumption and (2) stochastic effects 
Table 3

Ages and Masses of the FUV-defined SF Regions Assuming Different Extinction Curves

\begin{tabular}{|c|c|c|c|c|c|c|c|}
\hline \multirow[t]{2}{*}{$\mathrm{ID}^{\mathrm{b}}$} & \multicolumn{2}{|c|}{ MW with $R v=3.1^{\mathrm{a}}$} & \multicolumn{2}{|c|}{$\mathrm{LMC}^{\mathrm{a}}$} & \multicolumn{2}{|c|}{$\mathrm{LMC}^{\mathrm{a}}$} & \multirow{2}{*}{$\begin{array}{c}\text { Extinction Curve } \\
\text { Adopted }^{\mathrm{c}}\end{array}$} \\
\hline & Age (Myr) & Mass $\left(10^{3} \mathcal{M}_{\odot}\right)$ & Age (Myr) & Mass $\left(10^{3} \mathcal{M}_{\odot}\right)$ & Age (Myr) & Mass $\left(10^{3} \mathcal{M}_{\odot}\right)$ & \\
\hline EB-FUV 1 & $186_{-26}^{+15}$ & $240 ._{-143}^{+355}$ & $47_{-34}^{+49}$ & $46 ._{-28}^{+74}$ & $3_{-3}^{+38}$ & $1.9_{-1.2}^{+3.4}$ & LMC \\
\hline EB-FUV 2 & $61_{-2}^{+1}$ & $7.7_{-3.2}^{+5.5}$ & $25_{-14}^{+20}$ & $2.7_{-1.2}^{+2.1}$ & $5_{-4}^{+22}$ & $0.4_{-0.2}^{+0.3}$ & Rv3.1 \\
\hline EB-FUV 3 & $28_{-7}^{+1}$ & $6.0_{-3.8}^{+15.9}$ & $5_{-5}^{+21}$ & $0.8_{-0.5}^{+2.2}$ & $2_{-2}^{+25}$ & $0.7_{-0.4}^{+2.1}$ & Rv3.1 \\
\hline EB-FUV 4 & $37_{-2}^{+1}$ & $18 ._{-7}^{+12}$ & $6_{-2}^{+12}$ & $1.7_{-0.8}^{+1.3}$ & $2_{-2}^{+3}$ & $1.3_{-0.6}^{+1.1}$ & LMC \\
\hline EB-FUV 5 & $56_{-5}^{+2}$ & $11_{-5}^{+10}$ & $11_{-7}^{+17}$ & $1.6_{-0.8}^{+1.5}$ & $2_{-2}^{+4}$ & $0.6_{-0.3}^{+0.6}$ & Rv3.1 \\
\hline EB-FUV 6 & $123_{-8}^{+1}$ & $560 ._{-278}^{+949}$ & $76_{-41}^{+45}$ & $320 ._{-166}^{+596}$ & $46_{-43}^{+75}$ & $180 ._{-96}^{+361}$ & SMC \\
\hline EB-FUV 7 & $71_{-8}^{+1}$ & $13 ._{-6}^{+46}$ & $44_{-40}^{+25}$ & $7.5_{-3.9}^{+30.2}$ & $19_{-19}^{+51}$ & $2.6_{-1.4}^{+11.6}$ & Rv3.1 \\
\hline EB-FUV 8 & $37_{-2}^{+1}$ & $8.8_{-4.7}^{+11.5}$ & $13_{-9}^{+23}$ & $2.4_{-1.3}^{+3.4}$ & $4_{-4}^{+32}$ & $0.6_{-0.4}^{+0.9}$ & Rv3.1 \\
\hline EB-FUV 9 & $8_{-3}^{+1}$ & $1.0_{-0.7}^{+2.1}$ & $\ldots$ & $\ldots$ & $\ldots$ & $\ldots$ & Rv3.1 \\
\hline EB-FUV 10 & $46_{-28}^{+17}$ & $666_{-53}^{+257}$ & $\cdots$ & $\cdots$ & $\ldots$ & $\ldots$ & Rv3.1 \\
\hline EB-FUV 11 & $51_{-3}^{+1}$ & $12 ._{-7}^{+15}$ & $16_{-12}^{+28}$ & $2.8_{-1.6}^{+4.0}$ & $3_{-3}^{+33}$ & $0.4_{-0.3}^{+0.7}$ & Rv3.1 \\
\hline EB-FUV 12 & $223_{-8}^{+6}$ & $1200_{-32}^{+42}$ & $90_{-20}^{+25}$ & $37 ._{-10}^{+14}$ & $26_{-16}^{+21}$ & $8.6_{-2.5}^{+3.5}$ & Rv3.1 \\
\hline EB-FUV 13 & $114_{-14}^{+2}$ & $48 ._{-29}^{+72 .}$ & $48_{-33}^{+44}$ & $18 ._{-11}^{+29}$ & $11_{-11}^{+63}$ & $3.0_{-1.9}^{+5.3}$ & LMC \\
\hline EB-FUV 14 & $53_{-9}^{+1}$ & $14_{-11}^{+42}$ & $11_{-10}^{+42}$ & $2.2_{-1.7}^{+7.2}$ & $2_{-2}^{+51}$ & $0.7_{-0.6}^{+2.7}$ & Rv3.1 \\
\hline EB-FUV 15 & $129_{-14}^{+1}$ & $260 ._{-164}^{+492}$ & $64_{-44}^{+64}$ & $120 ._{-76}^{+244}$ & $27_{-26}^{+101}$ & $43 ._{-29}^{+98}$ & Rv3.1 \\
\hline EB-FUV 16 & $129_{-34}^{+14}$ & $58 ._{-42}^{+154}$ & $30_{-27}^{+58}$ & $11_{-8}^{+33}$ & $2_{-2}^{+51}$ & $1.0_{-0.7}^{+3.2}$ & Rv3.1 \\
\hline EB-FUV 17 & $90_{-3}^{+2}$ & $16 ._{-5}^{+7}$ & $33_{-15}^{+13}$ & $5.0_{-1.7}^{+2.5}$ & $4_{-2}^{+10}$ & $0.4_{-0.2}^{+0.2}$ & Rv3.1 \\
\hline EB-FUV 18 & $67_{-1}^{+1}$ & $6.0_{-2.5}^{+6.7}$ & $46_{-28}^{+20}$ & $3.9_{-1.7}^{+4.9}$ & $27_{-24}^{+39}$ & $2.0_{-0.9}^{+2.8}$ & Rv3.1 \\
\hline EB-FUV 19 & $118_{-18}^{+5}$ & $330 ._{-204}^{+546}$ & $44_{-35}^{+45}$ & $100 ._{-68}^{+192}$ & $5_{-5}^{+58}$ & $7.6_{-5.1}^{+15.4}$ & LMC2 \\
\hline EB-FUV 20 & $54_{-5}^{+1}$ & $110 ._{-68}^{+217}$ & $22_{-18}^{+31}$ & $39 ._{-24}^{+81}$ & $5_{-5}^{+48}$ & $6.2_{-4.0}^{+14.3}$ & LMC2 \\
\hline EB-FUV 21 & $45_{-2}^{+1}$ & $4.6_{-2.1}^{+9.9}$ & $22_{-18}^{+22}$ & $2.0_{-0.9}^{+4.6}$ & $6_{-6}^{+38}$ & $0.4_{-0.2}^{+1.0}$ & Rv3.1 \\
\hline EB-FUV 22 & $40_{-4}^{+1}$ & $6.7_{-3.6}^{+12.6}$ & $15_{-12}^{+24}$ & $1.8_{-1.0}^{+3.9}$ & $4_{-4}^{+35}$ & $0.5_{-0.3}^{+1.1}$ & Rv3.1 \\
\hline EB-FUV 23 & $64_{-12}^{+4}$ & $43 ._{-29}^{+91}$ & $8_{-6}^{+36}$ & $3.7_{-2.6}^{+8.5}$ & $\ldots$ & $\ldots$ & Rv3.1 \\
\hline EB-FUV 24 & $46_{-2}^{+1}$ & $4.0_{-2.0}^{+5.9}$ & $22_{-17}^{+24}$ & $1.6_{-0.8}^{+2.6}$ & $6_{-6}^{+40}$ & $0.3_{-0.2}^{+0.5}$ & Rv3.1 \\
\hline EB-FUV 25 & $100_{-15}^{+2}$ & $410 ._{-290}^{+977}$ & $39_{-34}^{+53}$ & $140 ._{-101}^{+366}$ & $5_{-5}^{+78}$ & $11_{-8}^{+32}$ & Rv3.1 \\
\hline EB-FUV 26 & $64_{-8}^{+3}$ & $32 .+42$ & $11_{-8}^{+27}$ & $3.9_{-2.3}^{+5.5}$ & $\ldots$ & $\ldots$ & Rv3.1 \\
\hline EB-FUV 27 & $132_{-21}^{+4}$ & $1200 ._{-822}^{+2570}$ & $50_{-39}^{+66}$ & $400 ._{-280}^{+932}$ & $8_{-8}^{+88}$ & $45_{-33}^{+115}$ & LMC2 \\
\hline EB-FUV 28 & $44_{-2}^{+1}$ & $7.4_{-3.9}^{+11.0}$ & $18_{-13}^{+26}$ & $2.4_{-1.3}^{+3.9}$ & $5_{-5}^{+38}$ & $0.5_{-0.3}^{+0.9}$ & Rv3.1 \\
\hline EB-FUV 29 & $130_{-30}^{+6}$ & $820 ._{-610}^{+2381}$ & $45_{-40}^{+77}$ & $250 ._{-188}^{+795}$ & $5_{-5}^{+102}$ & $17 ._{-14}^{+62}$ & Rv3.1 \\
\hline EB-FUV 30 & $154_{-26}^{+1}$ & $8300_{-580}^{+2405}$ & $66_{-53}^{+89}$ & $310_{-224}^{+1011}$ & $20_{-20}^{+135}$ & $77 ._{-57}^{+277}$ & Rv3.1 \\
\hline EB-FUV 31 & $29_{-10}^{+3}$ & $18 ._{-12}^{+38}$ & $3_{-3}^{+12}$ & $1.4_{-1.0}^{+3.3}$ & $\ldots$ & $\ldots$ & Rv3.1 \\
\hline EB-FUV 32 & $17_{-1}^{+1}$ & $0.5_{-0.0}^{+0.1}$ & $17_{-3}^{+0}$ & $0.5_{-0.0}^{+0.1}$ & $17_{-6}^{+0}$ & $0.5_{-0.0}^{+0.1}$ & Rv3.1 \\
\hline EB-FUV 33 & $157_{-2}^{+1}$ & $28 ._{-10}^{+16}$ & $98_{-27}^{+39}$ & $16 ._{-6}^{+10}$ & $60_{-33}^{+55}$ & $9.0_{-3.6}^{+6.0}$ & Rv3.1 \\
\hline EB-FUV 34 & $109_{-16}^{+1}$ & $390_{-223}^{+1276}$ & $61_{-50}^{+47}$ & $200 ._{-118}^{+721}$ & $30_{-30}^{+77}$ & $90 ._{-55}^{+361}$ & Rv3.1 \\
\hline EB-FUV 35 & $244_{-44}^{+9}$ & $390 ._{-291}^{+1139}$ & $106_{-74}^{+136}$ & $130 ._{-99}^{+418}$ & $39_{-39}^{+194}$ & $400_{-31}^{+142}$ & Rv3.1 \\
\hline EB-FUV 36 & $143_{-10}^{+1}$ & $41_{-23}^{+70}$ & $80_{-42}^{+62}$ & $21_{-13}^{+39}$ & $44_{-41}^{+98}$ & $11_{-6}^{+21}$ & Rv3.1 \\
\hline EB-FUV 37 & $129_{-1}^{+1}$ & $8.9_{-0.7}^{+1.5}$ & $122_{-12}^{+6}$ & $8.4_{-0.7}^{+1.5}$ & $114_{-23}^{+14}$ & $7.8_{-0.6}^{+1.5}$ & Rv3.1 \\
\hline EB-FUV 38 & $36_{-16}^{+1}$ & $13 ._{-10}^{+54}$ & $4_{-4}^{+32}$ & $1.0_{-0.8}^{+5.0}$ & $\ldots$ & $\ldots$ & Rv3.1 \\
\hline EB-FUV 39 & $197_{-37}^{+7}$ & $1500 ._{-1179}^{+5370}$ & $77_{-65}^{+127}$ & $460 ._{-367}^{+1822}$ & $18_{-18}^{+185}$ & $83 ._{-68}^{+369}$ & Rv3.1 \\
\hline EB-FUV 40 & $28_{-3}^{+1}$ & $2.3_{-1.2}^{+3.9}$ & $7_{-5}^{+19}$ & $0.5_{-0.3}^{+0.9}$ & $3_{-3}^{+24}$ & $0.2_{-0.1}^{+0.4}$ & Rv3.1 \\
\hline EB-FUV 41 & $64_{-31}^{+20}$ & $38 ._{-33}^{+215}$ & $3_{-3}^{+40}$ & $1.3_{-1.2}^{+8.6}$ & $\ldots$ & $\ldots$ & Rv3.1 \\
\hline EB-FUV 42 & $303_{-16}^{+7}$ & $100 ._{-49}^{+97}$ & $168_{-68}^{+76}$ & $40 ._{-21}^{+42 .}$ & $73_{-54}^{+109}$ & $15 ._{-8}^{+17}$ & Rv3.1 \\
\hline EB-FUV 43 & $24_{-7}^{+4}$ & $6.0_{-3.9}^{+11.2}$ & $2_{-2}^{+4}$ & $0.8_{-0.5}^{+1.6}$ & $\ldots$ & $\ldots$ & Rv3.1 \\
\hline EB-FUV 44 & $51_{-1}^{+1}$ & $7.5_{-2.4}^{+3.5}$ & $25_{-10}^{+15}$ & $3.2_{-1.1}^{+1.6}$ & $6_{-3}^{+18}$ & $0.5_{-0.2}^{+0.3}$ & Rv3.1 \\
\hline EB-FUV 45 & $24_{-4}^{+2}$ & $1.7_{-0.8}^{+1.4}$ & $3_{-2}^{+3}$ & $0.2_{-0.1}^{+0.2}$ & $\ldots$ & $\ldots$ & Rv3.1 \\
\hline EB-FUV 46 & $84_{-9}^{+1}$ & $220 ._{-100}^{+711}$ & $54_{-47}^{+28}$ & $130 ._{-64}^{+485}$ & $33_{-33}^{+49}$ & $76_{-38}^{+305}$ & Rv3.1 \\
\hline EB-FUV 47 & $48_{-6}^{+1}$ & $7.4_{-4.9}^{+14.1}$ & $6_{-4}^{+31}$ & $0.6_{-0.4}^{+1.2}$ & $\ldots$ & $\ldots$ & Rv3.1 \\
\hline EB-FUV 48 & $60_{-1}^{+1}$ & $5.1_{-1.4}^{+1.8}$ & $48_{-8}^{+11}$ & $3.9_{-1.1}^{+1.5}$ & $39_{-22}^{+20}$ & $3.1_{-0.9}^{+1.3}$ & Rv3.1 \\
\hline EB-FUV 49 & $82_{-7}^{+2}$ & $12 ._{-7}^{+14}$ & $27_{-22}^{+27}$ & $3.5_{-1.9}^{+4.3}$ & $3_{-3}^{+30}$ & $0.3_{-0.2}^{+0.4}$ & Rv3.1 \\
\hline EB-FUV 50 & $374_{-26}^{+9}$ & $460 ._{-274}^{+672}$ & $221_{-99}^{+121}$ & $200 ._{-123}^{+321}$ & $107_{-85}^{+192}$ & $75_{-48}^{+132}$ & Rv3.1 \\
\hline EB-FUV 51 & $119_{-33}^{+2}$ & $344_{-25}^{+159}$ & $46_{-43}^{+75}$ & $111_{-9}^{+60}$ & $6_{-6}^{+115}$ & $0.9_{-0.7}^{+5.2}$ & Rv3.1 \\
\hline EB-FUV 52 & $226_{-17}^{+8}$ & $130 ._{-65}^{+129}$ & $100_{-42}^{+64}$ & $46 .+49$ & $39_{-34}^{+63}$ & $15 ._{-8}^{+17}$ & LMC \\
\hline
\end{tabular}


Table 3

(Continued)

\begin{tabular}{|c|c|c|c|c|c|c|c|}
\hline \multirow[t]{2}{*}{$\mathrm{ID}^{\mathrm{b}}$} & \multicolumn{2}{|c|}{ MW with $R v=3.1^{\mathrm{a}}$} & \multicolumn{2}{|c|}{$\mathrm{LMC}^{\mathrm{a}}$} & \multicolumn{2}{|c|}{$\mathrm{LMC}^{\mathrm{a}}$} & \multirow{2}{*}{$\begin{array}{c}\text { Extinction Curve } \\
\text { Adopted }^{\mathrm{c}} \\
\end{array}$} \\
\hline & Age (Myr) & $\operatorname{Mass}\left(10^{3} \mathcal{M}_{\odot}\right)$ & Age (Myr) & Mass $\left(10^{3} \mathcal{M}_{\odot}\right)$ & Age (Myr) & Mass $\left(10^{3} \mathcal{M}_{\odot}\right)$ & \\
\hline EB-FUV 53 & $313_{-13}^{+7}$ & $80 .+37$ & $175_{-53}^{+60}$ & $32+25$ & $78_{-43}^{+84}$ & $12 ._{-5}^{+10}$ & Rv3.1 \\
\hline EB-FUV 54 & $113_{-17}^{+4}$ & $34 ._{-22}^{+64}$ & $44_{-36}^{+49}$ & $11 ._{-8}^{+24}$ & $5_{-5}^{+69}$ & $0.9_{-0.6}^{+2.0}$ & Rv3.1 \\
\hline EB-FUV 55 & $123_{-10}^{+1}$ & $15 ._{-9}^{+25}$ & $67_{-39}^{+54}$ & $7.6_{-4.5}^{+14.2}$ & $36_{-34}^{+85}$ & $3.7_{-2.3}^{+7.6}$ & Rv3.1 \\
\hline EB-FUV 56 & $156_{-6}^{+1}$ & $68 ._{-33}^{+67}$ & $88_{-36}^{+54}$ & $35 ._{-18}^{+37}$ & $48_{-41}^{+80}$ & $17 ._{-9}^{+20}$ & Rv3.1 \\
\hline EB-FUV 57 & $64_{-8}^{+1}$ & $440 ._{-288}^{+1041}$ & $25_{-21}^{+38}$ & $140 ._{-98}^{+379}$ & $5_{-5}^{+58}$ & $19 ._{-13}^{+55}$ & LMC2 \\
\hline EB-FUV 58 & $143_{-7}^{+1}$ & $95 ._{-50}^{+123}$ & $84_{-39}^{+57}$ & $52 .+74$ & $48_{-43}^{+94}$ & $27 ._{-15}^{+41}$ & Rv3.1 \\
\hline EB-FUV 59 & $97_{-11}^{+1}$ & $6.0_{-2.2}^{+28.3}$ & $71_{-63}^{+25}$ & $4.1_{-1.6}^{+22.1}$ & $50_{-50}^{+46}$ & $2.7_{-1.1}^{+16.6}$ & Rv3.1 \\
\hline EB-FUV 60 & $84_{-4}^{+1}$ & $150 ._{-76}^{+230}$ & $51_{-33}^{+32}$ & $87 ._{-45}^{+142}$ & $27_{-25}^{+56}$ & $41_{-22}^{+72}$ & Rv3.1 \\
\hline EB-FUV 61 & $184_{-12}^{+1}$ & $11_{-6}^{+24}$ & $124_{-73}^{+59}$ & $6.6_{-3.4}^{+15.4}$ & $74_{-69}^{+108}$ & $3.6_{-2.0}^{+9.4}$ & Rv3.1 \\
\hline EB-FUV 62 & $31_{-1}^{+1}$ & $3.3_{-0.5}^{+0.5}$ & $17_{-3}^{+3}$ & $1.5_{-0.2}^{+0.3}$ & $8_{-3}^{+7}$ & $0.6_{-0.1}^{+0.1}$ & LMC \\
\hline EB-FUV 63 & $110_{-2}^{+1}$ & $7.5_{-3.1}^{+5.3}$ & $76_{-25}^{+32}$ & $4.8_{-2.1}^{+3.7}$ & $50_{-34}^{+57}$ & $3.0_{-1.3}^{+2.5}$ & Rv3.1 \\
\hline EB-FUV 64 & $16_{-10}^{+1}$ & $1.7_{-1.3}^{+11.7}$ & $3_{-3}^{+13}$ & $0.3_{-0.3}^{+2.6}$ & $\cdots$ & $\ldots$ & Rv3.1 \\
\hline EB-FUV 65 & $47_{-2}^{+1}$ & $8.6_{-4.3}^{+8.5}$ & $18_{-12}^{+24}$ & $2.6_{-1.4}^{+2.8}$ & $5_{-5}^{+31}$ & $0.5_{-0.3}^{+0.6}$ & Rv3.1 \\
\hline EB-FUV 66 & $157_{-6}^{+1}$ & $250 ._{-65}^{+590}$ & $130_{-77}^{+25}$ & $200 ._{-55}^{+516}$ & $101_{-94}^{+54}$ & $150 ._{-44}^{+439}$ & LMC2 \\
\hline EB-FUV 67 & $21_{-1}^{+1}$ & $1.0_{-0.2}^{+0.7}$ & $16_{-10}^{+4}$ & $0.7_{-0.1}^{+0.5}$ & $11_{-8}^{+9}$ & $0.4_{-0.1}^{+0.4}$ & Rv3.1 \\
\hline EB-FUV 68 & $2_{-2}^{+1}$ & $0.5_{-0.4}^{+1.9}$ & $\ldots$ & $\ldots$ & $\cdots$ & $\cdots$ & Rv3.1 \\
\hline EB-FUV 69 & $45_{-7}^{+1}$ & $6.8_{-4.6}^{+14.5}$ & $6_{-4}^{+31}$ & $0.5_{-0.4}^{+1.2}$ & $\ldots$ & $\ldots$ & Rv3.1 \\
\hline EB-FUV 70 & $39_{-1}^{+1}$ & $2.3_{-0.0}^{+2.7}$ & $39_{-24}^{+0}$ & $2.3_{-0.0}^{+2.9}$ & $39_{-35}^{+0}$ & $2.3_{-0.0}^{+3.2}$ & Rv3.1 \\
\hline EB-FUV 71 & $40_{-5}^{+1}$ & $144_{-9}^{+26}$ & $9_{-6}^{+30}$ & $2.1_{-1.4}^{+4.4}$ & $3_{-3}^{+36}$ & $0.8_{-0.5}^{+1.8}$ & Rv3.1 \\
\hline EB-FUV 72 & $49_{-2}^{+1}$ & $8.5_{-3.9}^{+7.1}$ & $16_{-11}^{+21}$ & $2.2_{-1.0}^{+2.0}$ & $3_{-3}^{+17}$ & $0.4_{-0.2}^{+0.4}$ & Rv3.1 \\
\hline EB-FUV 73 & $70_{-1}^{+1}$ & $8.5_{-2.2}^{+3.0}$ & $57_{-11}^{+12}$ & $6.6_{-1.8}^{+2.5}$ & $46_{-22}^{+24}$ & $5.1_{-1.5}^{+2.1}$ & Rv3.1 \\
\hline EB-FUV 74 & $123_{-2}^{+1}$ & $14 ._{-6}^{+10 .}$ & $84_{-27}^{+37}$ & $9.0_{-3.9}^{+6.8}$ & $56_{-38}^{+65}$ & $5.6_{-2.5}^{+4.6}$ & Rv3.1 \\
\hline EB-FUV 75 & $32_{-6}^{+1}$ & $93 ._{-42}^{+331}$ & $13_{-11}^{+17}$ & $300_{-14}^{+120}$ & $5_{-5}^{+25}$ & $8.9_{-4.4}^{+40.2}$ & LMC2 \\
\hline EB-FUV 76 & $116_{-14}^{+1}$ & $15_{-5}^{+57}$ & $84_{-67}^{+30}$ & $10 ._{-4}^{+45}$ & $60_{-60}^{+54}$ & $6.8_{-2.7}^{+33.7}$ & Rv3.1 \\
\hline EB-FUV 77 & $6_{-1}^{+1}$ & $0.2_{-0.0}^{+1.1}$ & $5_{-5}^{+0}$ & $0.2_{-0.0}^{+1.2}$ & $5_{-5}^{+0}$ & $0.2_{-0.0}^{+1.3}$ & Rv3.1 \\
\hline
\end{tabular}

Notes.

${ }^{a}$ For all sources, a foreground extinction of $E(B-V)=0.22$ was applied with MW-type dust, and the additional internal extinction (from the total $E(B-V)$ given in Column 9 of Table 1) using three different dust types: "Rv3.1" stands for average MW extinction with $R v=3.1$ (Cardelli et al. 1989); "LMC" indicates the average LMC extinction for stars outside LMC2 by Misselt et al. (1999); "LMC2" indicates that the extinction curve by Misselt et al. (1999) was used.

${ }^{\mathrm{b}}$ Corresponding to the IDs in Table 1 and the blue labels in Figure 1.

${ }^{\mathrm{c}}$ The results for the extinction curve given in this column were adopted in the analysis. Only for source 6, we adopted an SMC-type extinction curve, which gives age $=4_{-4}^{+118} \mathrm{Myr}$ and mass $=11_{-8}^{+43} \times 10^{3} \mathcal{M}_{\odot}$.

from the top-IMF small number statistics. The latter affects both the analysis of integrated measurements and of resolved stellar counts. However, it affects only the small mass clusters. As we explained above, we restricted our analysis sample so to include sources with $\geqslant 5$ massive stars, in order to minimize problems of stochasticity. More importantly, we do not interpret our results in terms of mass distributions of individual clusters; instead, we add the masses of SF regions in broad age bins (next section) in order to obtain the total stellar mass formed at different epochs. In this way, we derive information on global star formation with broad time resolution, and stochastic effects on individual cluster masses average out. As for the assumption of "SSP" (or instantaneous star formation of each region), we tested the results by also using models with exponential SFH, decaying over short timescales: the results showed no appreciable difference. The measured FUV-NUV color of most sources is incompatible with CSP ("continuous star formation stellar populations") model colors of any age, ruling out the CSP assumption often used to derive global galaxy SFR, as not applicable to the individual SF regions in our sample. Strict coevality is not observed even in bona fide globular clusters, the epitome of "single age" stellar population, therefore some degree of uncertainty is carried in all works by this assumption, which is, however, the most compatible with the observed properties of our young population sample.

\section{RESULTS AND DISCUSSION}

\subsection{Recent Star Formation from UV Fluxes}

The SF regions have ages $\lesssim 400 \mathrm{Myr}$, as derived in the previous section from UV photometry, due to the FUV selection, and their masses range from $2.0 \times 10^{2} \mathcal{M}_{\odot}$ to $1.5 \times 10^{6} \mathcal{M}_{\odot}$, when individual extinction correction is applied to the sources, as explained in Section 3.2.

We added the UV-derived masses of the SF regions in four age ranges to estimate the average SFR within these time intervals. We find: SFR $=1 \times 10^{-2} \mathcal{M}_{\odot} \mathrm{yr}^{-1}(2-10 \mathrm{Myr})$, SFR $=$ $1.5 \times 10^{-2} \mathcal{M}_{\odot} \mathrm{yr}^{-1}(10-100 \mathrm{Myr}), \mathrm{SFR}=4.4 \times 10^{-2} \mathcal{M}_{\odot} \mathrm{yr}^{-1}$ (100-200 Myr), and SFR $=1.4 \times 10^{-2} \mathcal{M}_{\odot} \mathrm{yr}^{-1}$ over the intire 2-100 Myr range. The results are shown in Figure 5; 


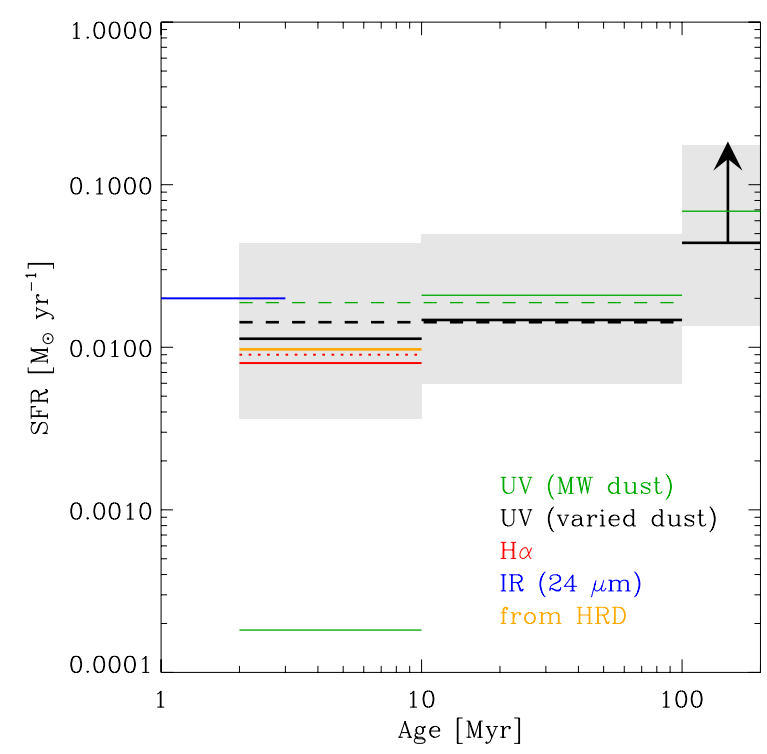

Figure 5. Average SFR of NGC 6822 over recent time intervals, derived by adding the masses of the FUV-defined SF regions within each epoch (solid lines; the dashed line shows the average over the wider range). The black lines show results obtained with reddening corrections as described in the text; gray boxes show the uncertainties, taking into account extinction spread within each region and photometric errors. Green lines show results obtained assuming MW-type $\left(R_{V}=3.1\right)$ extinction for all sources. The red lines show the SFR estimated from $\mathrm{H} \alpha$ in this paper (solid line) and by Hunter \& Elmegreen (2004) (dotted line). The blue line indicates the SFR derived from $24 \mu \mathrm{m}$ emission, and the yellow line shows the SFR derived from resolved stellar photometry of the youngest regions.

the uncertainties, shown as gray boxes, take into account the photometric errors and the $E(B-V)$ scatter within the SF regions, which is typically one order of magnitude larger than the photometric errors. The uncertainties are up to a factor of four of the derived values. Additional (smaller) uncertainties may arise from the assumption that the stellar population in each SF region has formed in a single burst, and from the adopted IMF. Coevality is supported by the H-R diagrams of seven young SF regions, studied with HST (Bianchi et al. 2001; Bianchi \& Efremova 2006), but it may be more questionable for older larger regions, which may result from the merging of subcomponents dissolving with time. Stochastic effects for low-mass complexes, as discussed previously, could affect individual masses, but average out when masses of several clusters are summed in broad age bins. Moreover, the total mass is dominated by the most massive SF regions.

Figure 5 illustrates also how the adopted type of interstellar extinction affects the results. It shows the SFR estimated using the extinction correction as derived in this work for individual regions, as well as by assuming that the total reddening for all sources is from Milky Way-type dust (green lines), as was adopted by, e.g., Wyder et al. (2007) and Hunter et al. (2010). The resulting SFR is significantly lower in the most recent epoch, because the most massive SF regions are shifted to older ages when we use this extinction curve.

For older ages, an incompleteness at low masses sets in, driven by our flux detection limit (see Figure 4). Our FUV-flux threshold translates (using our SSP models) into mass limits of $\sim 60 \mathcal{M}_{\odot}, \sim 170 \mathcal{M}_{\odot}$, and $2900 \mathcal{M}_{\odot}$ at $5 \mathrm{Myr}, 10 \mathrm{Myr}$, and $100 \mathrm{Myr}$, respectively, if only foreground extinction was present. The actual limit is higher since most young stellar populations have additional internal extinction. The average detection limit in the $2-10 \mathrm{Myr}$ bin is about $100 \mathcal{M}_{\odot}$. The cluster mass function derived by Lada \& Lada (2003) for embedded clusters in the solar vicinity has an exponent $\approx-2$ down to cluster masses of about $50 \mathcal{M}_{\odot}$, then it drops. If Lada \& Lada's results for solar vicinity were applicable to this dwarf irregular galaxy, the contribution of small mass clusters $\left(50-100 \mathcal{M}_{\odot}\right)$ to the total mass would be about $10 \%$ in this age bin, where we detect clusters with masses up to $4 \times 10^{4} \mathcal{M}_{\odot}$. However, star formation in NGC 6822 is patchy and may not resemble that of a massive spiral galaxy (Bianchi et al. 2001, 2010, 2011); in any case, this estimate should be regarded as an upper limit since the majority ( $\sim 90 \%)$ of the embedded clusters are expected to merge and become part of larger OB associations or field stars before they are 10 Myr old (Lada \& Lada 2003). At older ages, the mass function for embedded clusters is no longer applicable: according to Lada \& Lada (2003), after $100 \mathrm{Myr}$, 94\% of the embedded individual clusters have merged into large OB associations (if there was no cluster disruption, and if the above mass function were applicable, our detection limit would miss $45 \%$ of the clusters' mass at this epoch: this approximate figure can be taken as a conservative upper limit). The sum of the masses of our SF regions with ages between 100 and $200 \mathrm{Myr}$ yields SFR $\geqslant 0.044 \mathcal{M}_{\odot} \mathrm{yr}^{-1}$ in this period, in agreement with the study of stellar populations by Gallart et al. (1996), who found SFR $=0.04 \mathcal{M}_{\odot} \mathrm{yr}^{-1}$ in this epoch (adopting $E(B-V)$ $=0.24 \mathrm{mag}$ ). However, our value should be considered as a lower limit on the SFR because the FUV detection threshold corresponds to a high-mass detection limit for old populations. In addition, the uncertainty factors in the UV-based method, discussed previously, become significant at ages older than $\sim 100$ Myr.

We point out that our photometry of individual regions aims at isolating young SF complexes, and the older, diffuse galaxy population is subtracted from the flux. Therefore, it would not be appropriate to compare the total flux from the SF region photometry with integrated galaxy models assuming a global SFH (see also Section 2.3).

\subsection{The Very Recent Star Formation from $\mathrm{H \alpha}$ and Far-IR Measurements}

We also estimated the SFR of NGC6822 in very recent epochs from the $\mathrm{H} \alpha$ emission (Section 2.4). The total $\mathrm{H} \alpha$ flux from $\mathrm{H}$ II regions measured in this paper (the sum of the flux of the sources in Table 2) is $F_{\mathrm{H} \alpha}=1.8 \pm 0.2 \times 10^{-11} \mathrm{erg} \mathrm{s}^{-1} \mathrm{~cm}^{-2}$. After correcting the flux of the individual sources for reddening, using $E(B-V)$ values from the associated or nearest FUV sources, and $A_{\mathrm{H} \alpha} / E(B-V)=2.5$, the total unreddened flux is $F_{\mathrm{H} \alpha}$ (unreddened) $=4 \pm 1 \times 10^{-11} \mathrm{erg} \mathrm{s}^{-1} \mathrm{~cm}^{-2}$, corresponding to a luminosity of $\log L_{\mathrm{H} \alpha}=39.1 \pm 0.2 \mathrm{erg} \mathrm{s}^{-1}$. The uncertainty takes into account photometric errors and $E(B-V)$ scatter within individual SF regions. Most of the $\mathrm{H} \alpha$ emission $(\sim 70 \%)$ comes from $\mathrm{H} \alpha$ regions 5, 15, and 26 (see Figure 1 and Table 2). These include the $\mathrm{HII}$ regions Hubble V and Hubble $\mathrm{X}$, where an excellent agreement was found (under the assumption of optically thick gas) between the $\mathrm{H} \alpha$ luminosity and the number of ionizing photons estimated using resolved HST photometry (Bianchi et al. 2001; Bianchi \& Efremova 2006) and VLT spectroscopy (B. V. Efremova et al. 2011, in preparation). The $\mathrm{H} \alpha$ luminosity translates into SFR = $0.008 \pm 0.003 \mathcal{M}_{\odot} \mathrm{yr}^{-1}$ using the calibration by Panuzzo et al. (2003), which is based on the same SSP models we used to analyze the UV fluxes, the difference between the case (models) with and without dust being less than the uncertainty. Using other calibrations, we obtain similar results: for example, $S F R=$ 
$0.01 \pm 0.003 \mathcal{M}_{\odot} \mathrm{yr}^{-1}$ if we use the calibration by Hirashita et al. (2003), with $f=1$. Among our $\mathrm{H} \alpha$-defined H II regions, there is one (FUV source EB-FUV 52, $\mathrm{H} \alpha$ source EB-H $\alpha 25$ ) which includes only two blue stars (an O-type star and an early B-type supergiant, according to our VLT spectroscopy). This gives an indication of the sensitivity of our $\mathrm{H} \alpha$ source detection. In general, $\mathrm{H} \alpha$-based SFRs should always be regarded as a lower limit, due to the possibility of leakage of ionizing photons.

$\mathrm{H} \alpha$ emission traces the hottest, most massive stars, and this estimate is in good agreement with the UV-derived SFR in the recent 2-10 Myr, as we would expect. Our result is also in agreement with previous estimates of SFR based on $\mathrm{H} \alpha$ luminosity: $\mathrm{SFR}=0.01 \mathcal{M}_{\odot} \mathrm{yr}^{-1}$ by Hunter $\&$ Elmegreen (2004), and SFR $=0.016 \mathcal{M}_{\odot} \mathrm{yr}^{-1}$ by Cannon et al. (2006).

Star formation more recent than 1-2 Myr is embedded in dust and detectable by $24 \mu \mathrm{m}$ dust emission rather than in the UV, where the stellar flux is still heavily obscured. We estimated the SFR from the $24 \mu \mathrm{m}$ emission using the Spitzer/ MIPS measurements by Cannon et al. (2006), and the second term in Equation (D10) of Leroy et al. (2008). We derived $\operatorname{SFR}(24 \mu \mathrm{m})=0.02 \mathcal{M}_{\odot} \mathrm{yr}^{-1}$; this value is shown with a blue line in Figure 5, over a very short time interval, since IR dust emission typically traces the youngest populations, where the massive stars have not yet dissipated the dust of the parent cloud. Therefore, the far-IR detected star formation should complement the stellar mass of young populations detected from FUV measurements.

\section{SUMMARY AND CONCLUSIONS}

We have defined regions of recent star formation in NGC 6822 from GALEX wide-field FUV imaging, and derived ages and masses from their FUV, NUV photometry compared with SSP model populations. UV light is a good tracer of stellar populations up to a few hundred million years old. Extinction by interstellar dust has been estimated in each SF region from resolved photometry of the stars it contains: with an average value of $E(B-V)=0.37 \mathrm{mag}$, it exceeds in most cases the foreground reddening of $E(B-V)=0.22 \mathrm{mag}$. The characteristics of the internal (within NGC6822) selective extinction at UV wavelengths have been explored by comparing results from integrated UV photometry with H-R diagrams from high-resolution stellar photometry available for a number of well-studied SF regions. We found that a UV-steep, non-MW-type extinction is preferable for high surface brightness SF regions and adopt it for sources with surface brightness $<25 \mathrm{mag} \operatorname{arcsec}^{-2}$. MWtype extinction with $R_{V}=3.1$ seems adequate for less compact regions, which generally tend to be older. Such ad hoc criterion, suggested by our study for this particular case, will be verified over a larger sample of Local Group galaxies with new multiband HST imaging (e.g., Bianchi et al. 2010, 2011). This study has shown quantitatively that large variations of dust characteristics as a function of environment, and related to star formation intensity, exist within a single galaxy. The significant effect that the extinction correction bears on SFR derived from UV fluxes (Figure 5) highlights the limitations of integrated SFR recipes, if internal extinction is not properly accounted for.

We avoided commonly used methods for deriving $E(B-V)$ from the ratio of FUV to $24 \mu \mathrm{m}$ fluxes (e.g., Burgarella et al. 2005), because most far-IR emitting regions in this galaxy are clearly not co-located with the FUV emitting regions (e.g., Bianchi 2007). UV and far-IR bands trace different populations, and using the flux ratio would consequently overcorrect the

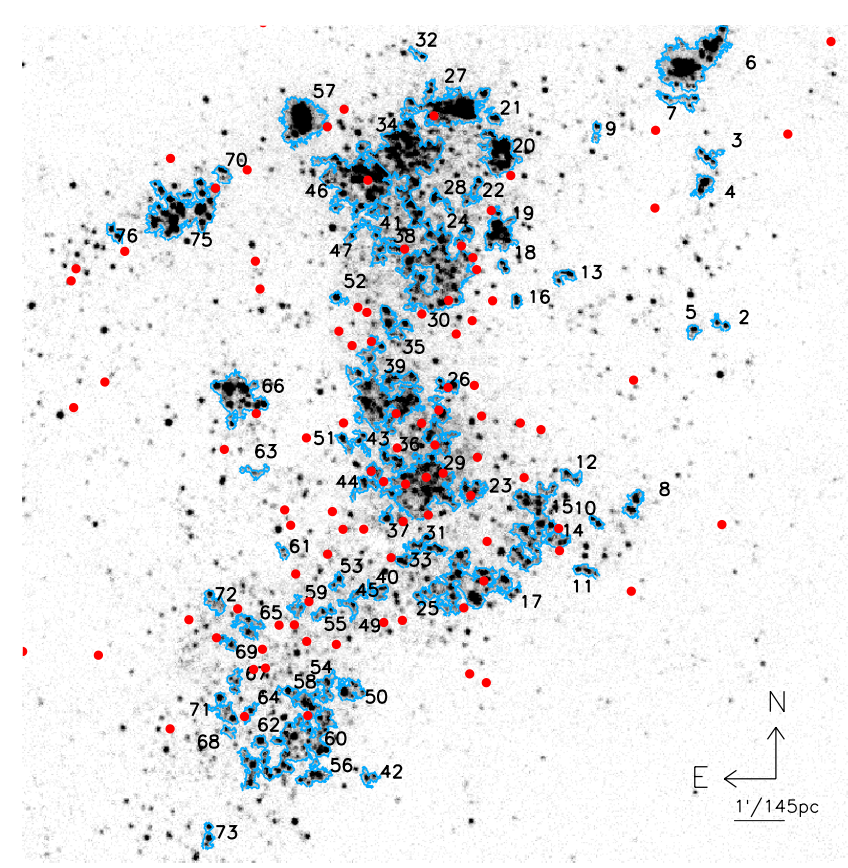

Figure 6. Distribution of FUV-defined SF regions (blue contours) compared with the location of known Cepheids in NGC 6822 from the catalog of Pietrzyński et al. (2004) (red dots), shown over the GALEX FUV image.

FUV fluxes. As can be seen in the $24 \mu \mathrm{m}$ Spitzer image of NGC 6822 published by Cannon et al. (2006), most peaks of IR emission trace $\mathrm{H} \alpha$ emission sites, with the exception of their region 11 , which is not a source of enhanced $\mathrm{H} \alpha$ emission. The $24 \mu \mathrm{m}$ emission originates from dust heated by newly formed stars, still embedded in their parental clouds. The FUVbright regions are more uniformly spread, because populations older than $\sim 10 \mathrm{Myr}$, no longer associated with either dust or significantly ionizing gas, still emit detectable FUV flux; only the youngest, most compact regions are bright in both FUV and $\mathrm{H} \alpha$.

We estimated the total stellar mass formed in recent time intervals by summing the masses of individual SF regions of corresponding ages. We derive an average SFR $=1.4 \times$ $10^{-2} \mathcal{M}_{\odot} \mathrm{yr}^{-1}$ over the past $100 \mathrm{Myr}$. For older ages, the FUV detection becomes incomplete and our method less robust, due to dissolving and possible merging of aging SF complexes. The uncertainty on SFR due to photometric errors and extinction correction (the major factor) is very large (shown in Figure 5). The overall level of star formation activity may not be significantly variable in the last $100 \mathrm{Myr}$, if we add to the FUV-detected young populations the embedded star formation component, traced by far-IR emission from the dust which extinguishes the FUV flux. We found, similarly to Kang et al. (2009), that the H $\alpha$ SFR estimate is a good measurement of the recent $(<10 \mathrm{Myr})$ star formation as assessed by the FUV imaging, when it is concentrated in bright compact sources, which are likely to be optically thick.

Finally, we examine the location of the known Cepheids in NGC 6822 using the catalog by Pietrzyński et al. (2004). Such stars are the evolved descendants of populations formed mostly in earlier epochs than those sampled by this work. A few are within our FUV-source contours, but mostly they avoid the FUV-bright regions and follow instead the optical appearance of the galaxy (Figure 6). While the youngest, FUV-bright SF regions are mostly found in the northern third of the galaxy, the Cepheids populate more uniformly the middle and southern 
part of the galaxy. We estimated their ages from the period-age relation derived by Efremov (2003) for Cepheids of similar metallicity (in the LMC). According to this relation, $85 \%$ of the Cepheids in NGC 6822 are older than 70 Myr. However, the period-age relation is constrained by very few data points at young ages (see Figure 3 of Efremov 2003), and we consider the overall spatial distribution more informative than individual ages.

We thank Philip Massey for very helpful clarifications on the calibration of the $\mathrm{H} \alpha$ image, Alin Tolea for initial discussions about the source-contour definition, and the anonymous referee for valuable comments.

The GALEX data presented in this paper were obtained from the Multimission Archive at the Space Telescope Science Institute (MAST). STScI is operated by the Association of Universities for Research in Astronomy, Inc., under NASA contract NAS5-26555. Support for MAST for non-HST data is provided by the NASA Office of Space Science via grant NAG57584 and by other grants and contracts. GALEX (The Galaxy Evolution Explorer) is a NASA Small Explorer, launched in 2003 April. We gratefully acknowledge NASA's support for construction, operation, and science analysis of the GALEX mission, developed in cooperation with the Centre National d'Etudes Spatiales of France and the Korean Ministry of Science and Technology. The $\mathrm{H} \alpha$ image used in this paper was obtained by Massey et al. (2007a) as part of the Survey of Local Group Galaxies Currently Forming Stars and downloaded from http://www.archive.noao.edu/nsa/. S.-C.R. is supported by the NRF of Korea to the Center for Galaxy Evolution Research.

\section{REFERENCES}

Bianchi, L. 2007, in UV Astronomy: Stars from Birth to Death, ed. A. I. Gomez de Castro \& M. Barstow (Madrid: Editorial Complutense), 65

Bianchi, L. 2009, Ap\&SS, 320, 11

Bianchi, L. 2011, Astrophys. Space Sci., in press

Bianchi, L., Chandar, R., \& Ford, H. 1999, Mem. Soc. Astron. Ital., 70, 629

Bianchi, L., \& Efremova, B. V. 2006, AJ, 132, 378

Bianchi, L., Kang, Y.-B., Efremova, B., Thilker, D., Hodge, P., Massey, P., \& Olsen, K. 2011, Astrophys. Space Sci., in press

Bianchi, L., Madore, B., Thilker, D., Gil de Paz, A., \& GALEX Science Team, 2003a, BAAS, 35, 1354

Bianchi, L., Madore, B., Thilker, D., Gil de Paz, A., Martin, C., \& The GALEX Team, 2003b, in The Local Group as an Astrophysical Laboratory, ed. M. Livio \& T. Brown (Baltimore, MD: STScI), 10

Bianchi, L., Scuderi, S., Massey, P., \& Romaniello, M. 2001, AJ, 121, 2020

Bianchi, L., et al. 2010, A\&AS, 215, 455.25
Boissier, S., et al. 2007, ApJS, 173, 524

Burgarella, D., Buat, V., \& Iglesias-Pramo, J. 2005, MNRAS, 360, 1413

Calzetti, D. 2001, PASP, 113, 1449

Calzetti, D., et al. 2005, ApJ, 633, 871

Cannon, J. M., et al. 2006, ApJ, 652, 1170

Cardelli, J. A., Clayton, G. C., \& Mathis, J. S. 1989, ApJ, 345, 245

Chandar, R., Bianchi, L., Ford, H., \& Salasnich, B. 1999, PASP, 111, 794

Cortese, L., et al. 2006, ApJ, 637, 242

de Blok, W. J. G., \& Walter, F. 2006, AJ, 131, 343

Efremov, Y. N. 2003, Astron. Rep., 47, 1000

Fall, S. M., Chandar, R., \& Whitmore, B. C. 2009, ApJ, 704, 453

Fatuzzo, M., \& Adams, F. C. 2008, ApJ, 675, 1361

Gallart, C., Aparicio, A., Bertelli, G., \& Chiosi, C. 1996, AJ, 112, 2596

Gil de Paz, A., et al. 2007, ApJS, 173, 185

Girardi, L., Chiosi, C., Bertelli, G., \& Bressan, A. 1995, A\&A, 298, 87

Gordon, K. D., \& Clayton, G. C. 1998, ApJ, 500, 816

Hirashita, H., Buat, V., \& Inoue, A. K. 2003, A\&A, 410, 83

Hodge, P., Krienke, O. K., Bianchi, L., Massey, P., \& Olsen, K. 2010, PASP, 122,745

Hodge, P. W. 1977, ApJS, 33, 69

Hodge, P. W., Lee, M. G., \& Kennicutt, R. C., Jr. 1988, PASP, 100, 917

Hodge, P. W., Lee, M. G., \& Kennicutt, R. C., Jr. 1989, PASP, 101, 32

Hubble, E. P. 1925, ApJ, 62, 409

Hunter, D. A., \& Elmegreen, B. G. 2004, AJ, 128, 2170

Hunter, D. A., Elmegreen, B. G., \& Ludka, B. C. 2010, AJ, 139, 447

Kang, Y., Bianchi, L., \& Rey, S.-C. 2009, ApJ, 703, 614

Karachentsev, I. D., Karachentseva, V. E., Huchtmeier, W. K., \& Makarov, D. I 2004, AJ, 127, 2031

Kennicutt, R. C., Jr. 1998, ARA\&A, 36, 189

Killen, R. M., \& Dufour, R. J. 1982, PASP, 94, 444

Kinman, T. D., Green, J. R., \& Mahaffey, C. T. 1979, PASP, 91, 749

Kroupa, P. 2001, MNRAS, 322, 231

Lada, C. J., \& Lada, E. A. 2003, ARA\&A, 41, 57

Leroy, A. K., Walter, F., Brinks, E., Bigiel, F., de Blok, W. J. G., Madore, B., \& Thornley, M. D. 2008, AJ, 136, 2782

Martin, D. C., et al. 2005, ApJ, 619, L1

Massey, P., McNeill, R. T., Olsen, K. A. G., Hodge, P. W., Blaha, C., Jacoby, G. H., Smith, R. C., \& Strong, S. B. 2007a, AJ, 134, 2474

Massey, P., Olsen, K. A. G., Hodge, P. W., Jacoby, G. H., McNeill, R. T., Smith, R. C., \& Strong, S. B. 2007b, AJ, 133, 2393

McAlary, C. W., Madore, B. F., McGonegal, R., McLaren, R. A., \& Welch, D. L. 1983, ApJ, 273, 539

Melena, N. W., Elmegreen, B. G., Hunter, D. A., \& Zernow, L. 2009, AJ, 138, 1203

Meurer, G. R., et al. 2009, ApJ, 695, 765

Misselt, K. A., Clayton, G. C., \& Gordon, K. D. 1999, ApJ, 515, 128

Morrissey, P., et al. 2007, ApJS, 173, 682

Muschielok, B., et al. 1999, A\&A, 352, L40

O’Dell, C. R., Hodge, P. W., \& Kennicutt, R. C., Jr. 1999, PASP, 111, 1382

Pagel, B. E. J., Edmunds, M. G., \& Smith, G. 1980, MNRAS, 193, 219

Panuzzo, P., Bressan, A., Granato, G. L., Silva, L., \& Danese, L. 2003, A\&A, 409, 99

Pietrzyński, G., Gieren, W., Udalski, A., Bresolin, F., Kudritzki, R.-P., Soszyński, I., Szymański, M., \& Kubiak, M. 2004, AJ, 128, 2815

Venn, K. A., et al. 2001, ApJ, 547, 765

Wyder, T. K., et al. 2007, ApJS, 173, 293 